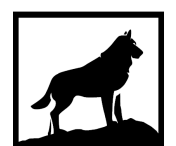

Michigan

Technological

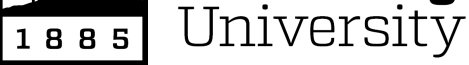

Michigan Technological University

Digital Commons @ Michigan Tech

2016

THE EFFECT OF SOLIDIFICATION RATE AND SOLUTIONIZING

QUENCH RATE ON THE MECHANICAL PROPERTIES AND

HARDENING RESPONSE OF ALUMINUM ALLOYS: A

QUANTITATIVE COMPARISON

Rafael Gil-Figueroa

Michigan Technological University, regilfig@mtu.edu

Copyright 2016 Rafael Gil-Figueroa

Recommended Citation

Gil-Figueroa, Rafael, "THE EFFECT OF SOLIDIFICATION RATE AND SOLUTIONIZING QUENCH RATE ON

THE MECHANICAL PROPERTIES AND HARDENING RESPONSE OF ALUMINUM ALLOYS: A QUANTITATIVE COMPARISON", Open Access Master's Thesis, Michigan Technological University, 2016.

https://doi.org/10.37099/mtu.dc.etdr/162

Follow this and additional works at: https://digitalcommons.mtu.edu/etdr

Part of the Metallurgy Commons 


\title{
THE EFFECT OF SOLIDIFICATION RATE AND SOLUTIONIZING QUENCH RATE ON THE MECHANICAL PROPERTIES AND HARDENING RESPONSE OF ALUMINUM ALLOYS: A QUANTITATIVE COMPARISON
}

\author{
By \\ Rafael E. Gil-Figueroa
}

\begin{abstract}
A THESIS
Submitted in partial fulfillment of the requirements for the degree of MASTER OF SCIENCE

In Materials Science and Engineering
\end{abstract}

MICHIGAN TECHNOLOGICAL UNIVERSITY

2016

(C) 2016 Rafael E. Gil-Figueroa 
This thesis has been approved in partial fulfillment of the requirements for the Degree of MASTER OF SCIENCE in Materials Science and Engineering.

Department of Materials Science and Engineering

Thesis Advisor: Dr. Paul Sanders

Committee Member: $\quad$ Dr. Greg Odegard

Committee Member: Dr. Adam Loukus

Committee Member: Dr. Walter Milligan

Department Chair: $\quad$ Dr. Stephen Kampe 


\section{Table of Contents}

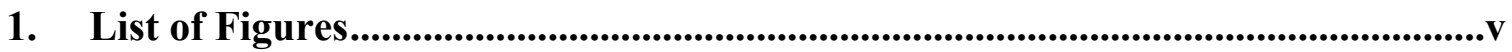

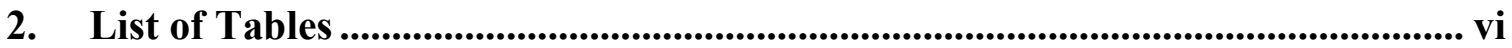

ACKNOWLEDGEMENT ........................................................................................... vii

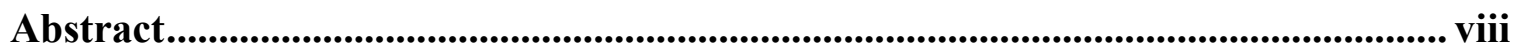

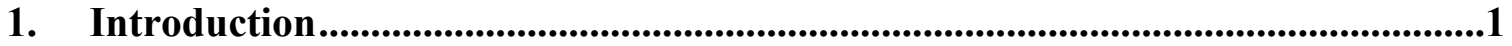

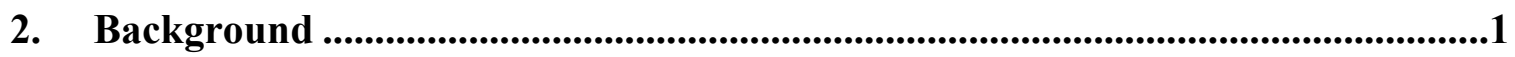

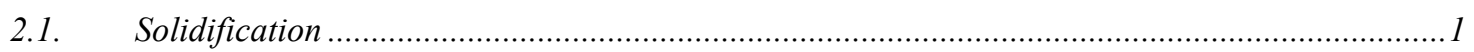

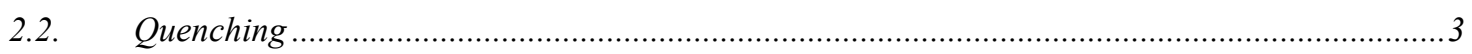

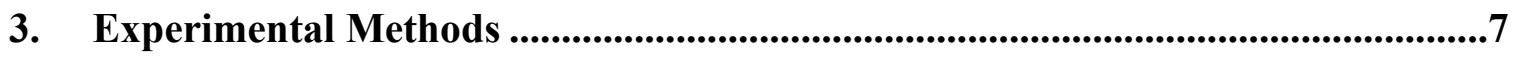

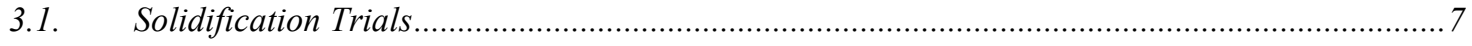

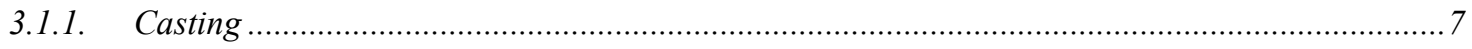

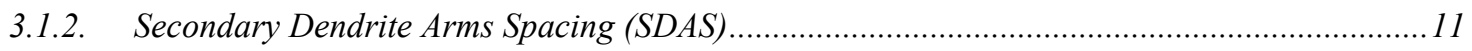

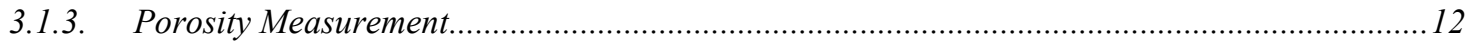

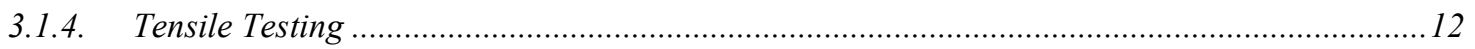

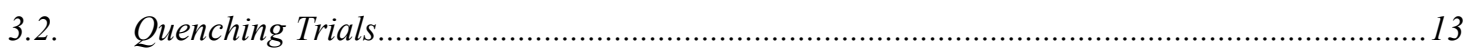

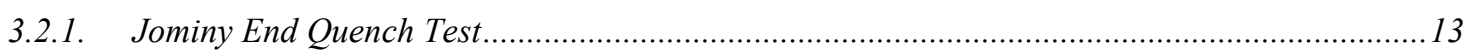

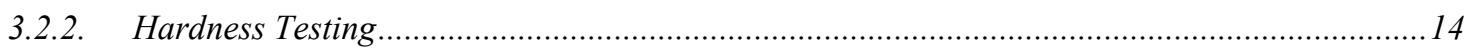

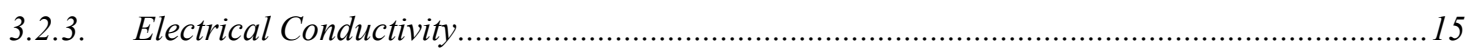

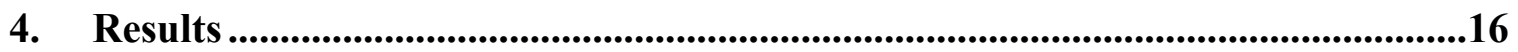

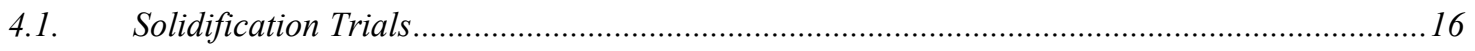

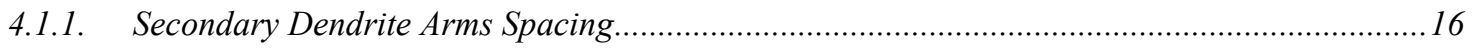

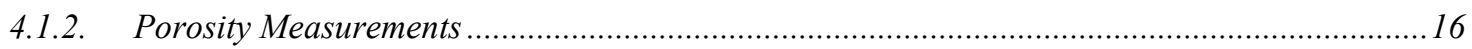

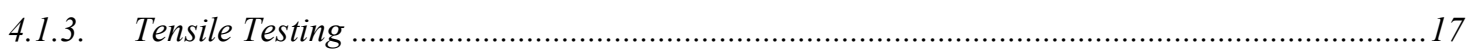

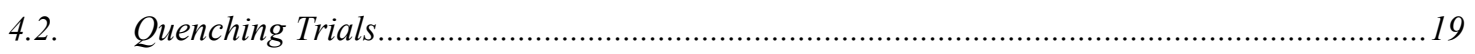

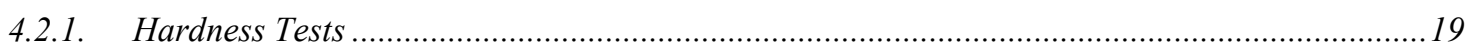

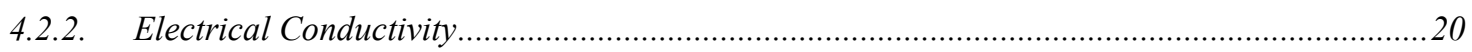

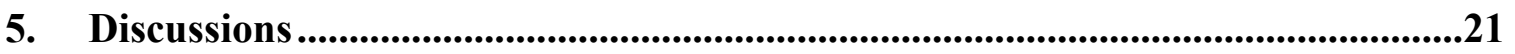

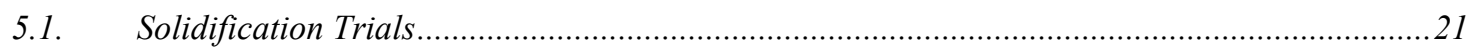

iii 


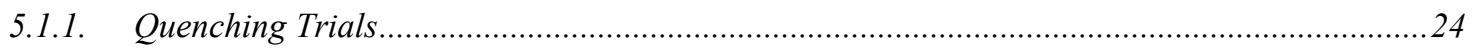

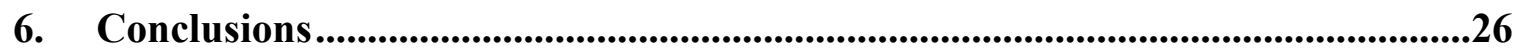

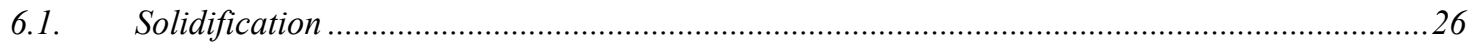

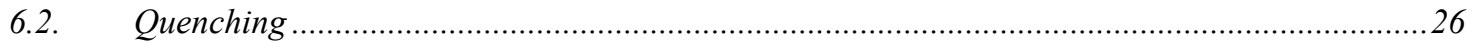

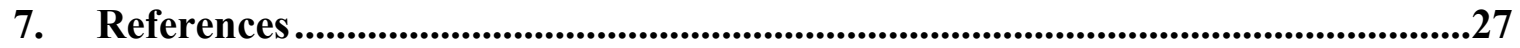

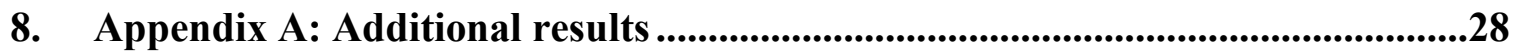

9. Appendix B: Selected SDAS images for all alloys................................................29 


\section{List of Figures}

Figure 2-1: Meyer hardness along a Jominy end quench bar of cast aluminum A356 [16]........................................................ 6 Figure 2-2: Hardness profile of 7075 and 7050 aluminum Jominy end quench specimens [14] ............................................ 7

Figure 3-1: JEQ bar being cut off of the runner before being sent to get machined to proper length ................................................ 8

Figure 3-2: First iteration of 3D printed JEQ bar casting that was used to create sand mold box. .................................................. 9

Figure 3-3: Finalized sand mold box, with second iteration 3D printed JEQ bar casting, also showing location features attached to the base plate.

Figure 3-4: Molds ready for casting, clamped into place, with the sand, chilled sand, and permanent mold aligned properly. Note grey

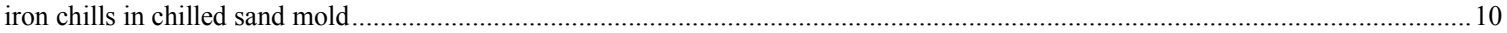

Figure 3-5: Sample of SDAS measurement image, with scale bar and line measuring distance from one dendrite to another, image is of

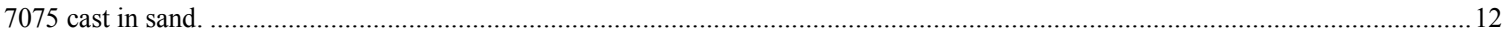
Figure 3-6: JEQ test in operation, with thermocouples at $0.5,1.5,2.5$, and 3.5 inches $(12.7,38.1,63.5$, and $88.9 \mathrm{~mm})$ along the length of the bar, corresponding to labels 1 through 4 , respectively. The ceramic washer is 0.146 inches $(3.7084 \mathrm{~mm})$ thick.......................14 Figure 3-7: Cast JEQ bar, pre-machining and before any testing has taken place ................................................................... 15 Figure 3-8: Schematic of hardness testing, with indents every $3.175 \mathrm{~mm}$ in the $\mathrm{x}$-direction and every $2 \mathrm{~mm}$ in the y-direction. .......... 15 Figure 4-1: Experimental SDAS averages from all the alloy/mold combinations with 95\% confidence shown. ...............................16 Figure 4-2: Porosity as of percentage difference between calculated density and referenced density from literature [20]................... 17 Figure 4-3: Stress-strain curves for A206, 7075, and 355 alloys, for all three mold types............................................................ 19 Figure 4-4: Hardness data down the length of the bar for A206, 7075 and 355 alloys in each mold type with 95\% confidence shown.20 Figure 4-5: Electrical conductivity down the length of the bar for A206, 7075 and 355 alloys, in each mold type with $95 \%$ confidence

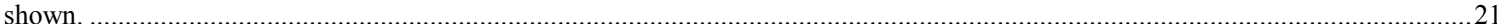

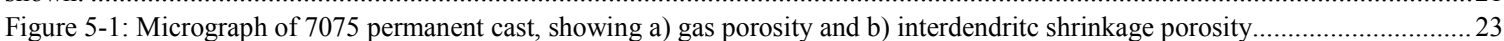
Figure 5-2: Statistical models for A206, 7075 and 319, based off of hardness as a function of distance .........................................25 Figure 9-1: A206 sand cast micrographs at 100x magnification, etched with Keller's Reagent, at two different locations...................29 Figure 9-2:A206 semi-permanent cast micrographs at 100x magnification, etched with Keller's Reagent, at two different locations... 30 Figure 9-3:A206 permanent cast micrographs at 100x magnification, etched with Keller's Reagent, at two different locations............ 31 Figure 9-4:: 7075 sand cast micrographs at 100x magnification, etched with Keller's Reagent, at two different locations.................. 32 Figure 9-5:7075 semi-permanent cast micrographs at 100x magnification, etched with Keller's Reagent, at two different locations.... 33 Figure 9-6:7075 permanent cast micrographs at 100x magnification, etched with Keller's Reagent, at two different locations ............34 Figure 9-7:319 semi-permanent cast micrographs at 100x magnification, etched with Keller's Reagent, at two different locations...... 35 Figure 9-8:319 permanent cast micrographs at 200x magnification etched with Keller's Reagent, at two different locations ................36 Figure 9-9:355 sand cast micrographs at 100x magnification etched with Keller's Reagent, at two different locations..........................37 Figure 9-10:355 semi-permanent cast micrographs at 100x magnification etched with Keller's Reagent, at two different locations..... 38 Figure 9-11:355 permanent cast micrographs at a) 100x magnification and b) 200x magnification etched with Keller's Reagent, at two

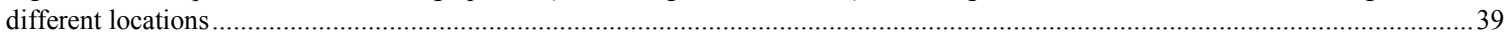
Figure 9-12:A356 sand cast micrographs at 100x magnification etched with Keller's Reagent at two different locations ...................40 Figure 9-13:A356 semi-permanent micrographs at 100x magnification etched with Keller's Reagent at two different locations..........41 Figure 9-14:A356 permanent cast micrographs at 200x magnification etched with Keller's Reagent, at two different locations...........42 


\section{List of Tables}

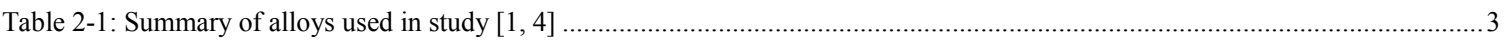

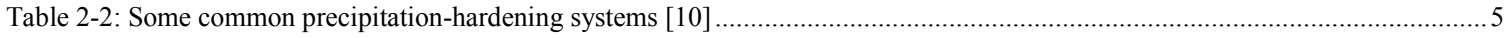

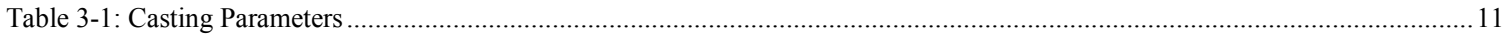

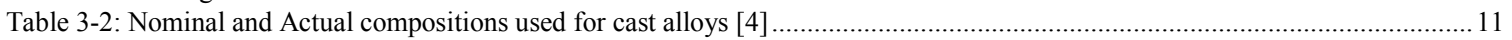

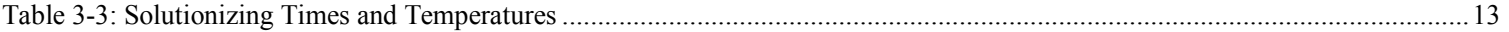

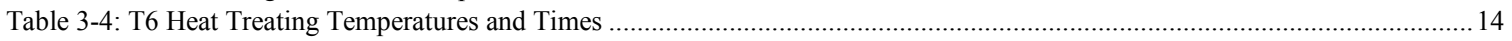

Table 4-1: Elastic Modulus, Yield Stress, Ultimate Stress, and Strain for Alloys cast............................................................... 18

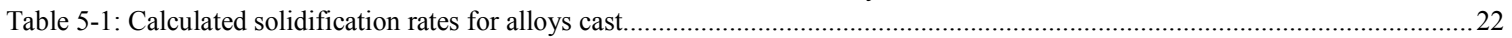

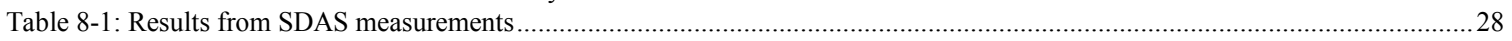

Table 8-2: Porosity of samples shown as a percentage difference between literary density and measured density [20] ......................28 


\section{ACKNOWLEDGEMENT}

I would first like to thank my thesis adviser Dr. Paul Sanders of the Materials Science and Engineering department at Michigan Technological University. His commentary has made me a better writer, his questions made me a better thinker and for that I will be forever grateful.

I would also like to thank both Southwestern Energy for sponsoring the project, and REL Inc. for being an amazing wellspring of knowledge. Without either one of these two companies, this work would not exist.

Finally, my journey here was not made alone and I would be remiss to not acknowledge my parents, my siblings and my beloved fiancé for all their support. I can say wholeheartedly that they have never doubted, and their faith in me has helped me accomplish this goal. I would also like to acknowledge my fellow graduate students in the department, for commiseration is done best with those who understand misery. Thank you all for your help, you were the 10,000 hands that carried me to this mountain top.

Rafael Eduardo Gil-Figueroa 


\begin{abstract}
A comparative study of five aluminum alloys was performed to characterize the effect of solidification rate and quench rate on casting microstructure and properties. The alloys were cast in the geometry for Jominy End Quench (JEQ) testing, so as to take advantage of the JEQ test's ability to give data on multiple quench rates in a single sample and illustrate the quench sensitivity of an alloy. While the Jominy End Quench test has been used in aluminum alloys, the effects of solidification rates have not been assessed in depth. The work done by other studies has either focused on a single alloy across multiple solidification rates, or on multiple alloys using a single solidification rate. To this end, three molds were created: a sand mold, a semi-permanent mold and a permanent mold, with the intent of casting JEQ bars out of multiple aluminum alloys for direct comparison. The tensile strength, hardness, porosity, and electrical conductivity were assessed, in an attempt to compare the quench sensitivities of the samples. This study will provide a starting point for more in-depth analyses of the alloys, i.e. the kinetics of precipitation strengthening over a range of length scales (cooling rates).
\end{abstract}




\section{Introduction}

The work herein focuses on two important aspects of aluminum alloy production, the solidification rate and the quench rate, both of which are time related. Both of these issues are related to heat flow out of a casting at two important junctions: the solidification of the casting and the quenching of the casting. This work was done to optimize the process cycle for a complex aluminum casting. The complexities of the casting cause issues when solidifying and quenching. The internal geometry of the casting is difficult to solidify quickly, as the cores of the mold do not remove heat quickly from the metal. The cores of the casting lose effectiveness in cooling the liquid metal, as the cores are surrounded on all sides by metal at the same temperature. Slow solidification rate will lead to poor mechanical properties, as primary phases will coarsen as the metal cools from liquid to solid. The internal complexities of the casting also cause quenching rates to decrease, as water has a more difficult path to travel in reaching the inner parts of the casting, allowing for quench induced precipitates to nucleate and grow while the water is delayed. This decreased quench rate will cause lessened mechanical properties, as quenched induced precipitates will grow instead of staying in solution. To simplify the process and pinpoint the important limits of the cooling rate, a test method usually used with steels was used in this scenario, the Jominy End Quench Test. The samples created for use with the Jominy End Quench Test were cast using three different molds, in an attempt to study the effects of solidification rate on mechanical properties.

\section{Background}

\subsection{Solidification}

Solidification rate controls many aspects of the mechanical properties of a cast piece, specifically the strength and elongation. Solidification rate will affect the size of any intermetallic phases, the spacing of dendrite arms, the size of the grains, and the morphology of the primary phase [1]. As Rundman stated "the process of solidification...is the defining event in the life cycle of a casting"[2]. When casting aluminum alloys, the faster solidification rates usually yield better strength as the smaller the grains will interrupt dislocation motion more effectively than large grains, due to Hall-Petch strengthening and a more finely distributed grain-boundary surface area $[1,3]$.

A downside to a faster solidification rate is the potential for interdendritic shrinkage porosity; if the metal solidifies faster than a given rate in the mold, the tensile forces created by the slowly contracting solid overpower the surface tension of the liquid still solidifying [1]. This leads to interdendritic shrinkage as the growing dendrites will pull apart, leaving voids between the grains. Another issue that may arise from faster solidification rates is hot tearing, where the thermally expanding mold pulls against the contracting solid metal. If the mold cannot change, then the forces created will tear the solid skin of metal [2]. The given rate is casting design related, as proper riser additions 
to the mold will mitigate both of these phenomenon, as well as proper mold design reducing stress concentrators like sharp corners [2].

Solidification rate also changes depending on the alloy in question; as the range between the liquidus and solidus, where the dendrites are actually formed, varies with respect to the composition of alloy [2]. The solidification rate is the change in temperature over the amount of time, with the time starting when the casting crosses the liquidus temperature and ends when the metal reaches the solidus temperature. In this study, five alloys were used, each with a varying array of important compositional elements, with the primary strengthening phase of the alloy or primary strengthening compositional elements of the alloy being used to define the alloy. Table 2-1 gives a summary of the alloys used in the study, with special attention being paid to the solidification range and the strengthening phases present.

A206 is an aluminum-copper alloy that has a liquidus temperature of $650^{\circ} \mathrm{C}$ and a solidus temperature of $570^{\circ} \mathrm{C}$, and the primary strengthening phase for the alloy is the theta phase, $\mathrm{Al}_{2} \mathrm{Cu}[1,4]$. The material also has been shown to retain a high tensile strength with high iron content (approximately $0.3-0.5 \%$ ) but only in the natural aged condition, T4. However, at any temper above T4, the elongation suffers, which is why the industry standard is T4 [5].

7075 is an aluminum-zinc-magnesium alloy that has a liquidus temperature of $635^{\circ} \mathrm{C}$ and a solidus temperature of $477^{\circ} \mathrm{C}$, and the primary strengthening phase for the alloy is the eta phase, $\mathrm{Zn}_{2} \mathrm{Mg}[1,4]$. The kinetics of this strengthening phase are such that 7075 is a very quench sensitive alloy, meaning that if there is a delay between the solutionizing and quenching of the part, or a slow quench, the mechanical properties will decrease sharply $[6,7] .7075$ is also very sensitive to iron impurities, as the intermetallic phases made with iron act as stress concentrators reducing the overall strength of the casting. Due to its high strength, 7075 is primarily used as an aerospace structural alloy $[4,7]$.

319 is an aluminum-silicon-copper that has a liquidus temperature of $605^{\circ} \mathrm{C}$ and a solidus temperature of $520^{\circ} \mathrm{C}$, and the primary strengthening phase for the alloy is the same as $\mathrm{A} 206$, the theta phase, $\mathrm{Al}_{2} \mathrm{Cu}[1,8]$. However, because of the presence of silicon, the strengthening also comes from the eutectic silicon. This would mean that the intermetallic phase of $\mathrm{Al}_{2} \mathrm{Cu}$ might negatively affect the mechanical properties, as the morphology of the intermetallic may act as a stress concentrator, though this depends on the morphology of the particles. 319 is also used as an automotive alloy, because of its good casting characteristics, heat treatability, multiple strengthening phases and good tolerance to iron [9].

355 is an aluminum-silicon-magnesium alloy that has a liquidus temperature of $620^{\circ} \mathrm{C}$ and a solidus temperature of $550^{\circ} \mathrm{C}$, and the primary strengthening phase for the alloy is the beta phase, $\mathrm{Mg}_{2} \mathrm{Si}$. [1, 10] The variant being used for the study uses a more tightly controlled composition within the 355 ranges for all the elements, with the goal of sticking more closely to the nominal composition limits for 355 . 
A356 is an aluminum-silicon-magnesium alloy that has a liquidus temperature of $615^{\circ} \mathrm{C}$ and a solidus temperature of $560^{\circ} \mathrm{C}$, and the primary strengthening phase for the alloy is the beta phase, $\mathrm{Mg}_{2} \mathrm{Si}$, present in the alloy $[1,4,11]$. The silicon present in the alloy can also strengthen the casting, but not in an unmodified state. Modification of the silicon is done using strontium additions to the alloy while melting. A356 is used as an automotive alloy, due to its excellent casting characteristics and corrosion resistance [1].

Table 2-1: Summary of alloys used in study [1, 4]

\begin{tabular}{|c|c|c|c|c|}
\hline Alloy & $\begin{array}{c}\text { Liquidus } \\
\text { Temperature } \\
\left({ }^{\mathbf{}} \mathbf{C}\right)\end{array}$ & $\begin{array}{c}\text { Solidus } \\
\text { Temperature } \\
\left({ }^{\mathbf{}} \mathbf{C}\right)\end{array}$ & $\begin{array}{c}\text { Temperature } \\
\text { Difference } \\
\left({ }^{\mathbf{0}} \mathbf{C}\right)\end{array}$ & $\begin{array}{c}\text { Key } \\
\text { Elements }\end{array}$ \\
\hline $\mathbf{A 2 0 6}$ & 650 & 570 & 80 & $\mathrm{Cu}$ \\
\hline $\mathbf{7 0 7 5}$ & 635 & 477 & 158 & $\mathrm{Zn}, \mathrm{Mg}$ \\
\hline $\mathbf{3 1 9}$ & 605 & 520 & 85 & $\mathrm{Si}, \mathrm{Cu}$ \\
\hline $\mathbf{3 5 5}$ & 620 & 550 & 70 & $\mathrm{Si}, \mathrm{Mg}$ \\
\hline $\mathbf{A 3 5 6}$ & 615 & 560 & 55 & $\mathrm{Si}, \mathrm{Mg}$ \\
\hline
\end{tabular}

Faster solidification positively effects the primary strengthening phases that might come out of solution during the initial solidification. By cooling faster from the liquid, the diffusion controlled formation of strengthening phases is limited, creating a more saturated solid solution and finer distribution of constituent particles, which are easier to heat treat $[1,10,12]$.

A wide solidification range presents challenges, as the wide gap will raise the potential for primary phases to coarsen instead of staying in solution. This will increase the solutionizing time, as the larger primary phases will need more energy to diffuse back into the matrix, as well as increase the likelihood for hot tearing and phase segregation.

\subsection{Quenching}

All of the alloys in this study are heat treatable, which means that they require a supersaturated solid solution. As Campbell states, "solid-state precipitation reactions are of great importance in engineering alloys....The reaction occurs when the initial phase composition (e.g., $\alpha_{0}, \beta_{0}$, or $\mathrm{I}_{0}$ ) transforms into a two-phase product that includes a new phase or precipitate" [10]. A three-step process is required for precipitation hardening to occur:

1. Solutionizing: Heating the material (in our case, aluminum) to a temperature such that the solute elements dissolve into the matrix (solution).

2. Quenching: Rapidly cooling the alloy to room temperature to trap the solute elements in the solid solution.

3. Aging: Heating the material to an intermediate temperature to nucleate and growth a finely dispersed precipitate phase out of the solid solution [10]. 
The mechanism for hardening is the precipitation of extremely fine particles [10]. These finely distributed particles that are dispersed throughout the matrix of the material are classified as particle hardening and act as a disruption to dislocation motion through the material at large. When the particles are grown initially, they are coherent within the matrix, which comes about from how they form out of the matrix. The supersaturate solid solution that is created during quenching will grow clusters of Guiner-Preston zones (GP). With the addition of more energy to the system, i.e. holding the system at an elevated temperature, those GP zones will grow into either the double prime or prime strengthening phase of the alloy. This elevated temperature promotes diffusion, which enables precipitation to occur. These double prime or prime phases tend to be transitional, but are usually the phase that will increase the strength of the alloy the most. This is due to the Orowan looping, where the small particles will have to cause dislocations to loop around them before the dislocations can continue through the material $[10,13]$. The best possible temper for this strengthening regime is the peak aged, T6. At this temper, the material has the optimal spacing between the particles. Adding more energy to the system will lead to the coarsening of these transitional phases to the stable phases, as staying at the elevated temperature for a longer time will increase the volume fraction and size of the particles past their most useful size[1,10]. Of all the alloy systems that can be precipitation hardened, aluminum alloys are one of the most important due to their high strength-to-weight ratio and other attractive mechanical properties. The most commonly used include the aluminum-copper series, the aluminumsilicon-magnesium series, and the aluminum-zinc series (Table 2-2) [10]. Table 2-2 give examples of all the growth regimes for the alloys, showing the transitional phases and the stable phase for each alloy, as well as give the shape of the precipitates. The shape of the precipitates is important for verification of how effective the quench was, as TEM is able to show the phases and their morphology. Slow or ineffective quenches change the morphology of the phases by allowing them to grow beyond the transitional stage. For many of the alloys shown in Table 2-2, the phase that gives the best mechanical properties is usually the prime phase, i.e. $\theta^{\prime}, \eta^{\prime}$, and $S^{\prime}[10]$. 
Table 2-2: Some common precipitation-hardening systems [10]

\begin{tabular}{|c|c|c|c|c|}
\hline Matrix & Solute & \multicolumn{2}{|c|}{ Transition structures(a) } & Equilibrium \\
\hline \multirow[t]{4}{*}{$\mathrm{Al}$} & $\mathrm{Cu}$ & (i) & $\begin{array}{l}\text { Platelike solute-rich } \\
\text { GP [1] zones }\end{array}$ & \multirow{4}{*}{$\theta-\mathrm{CuAl}_{2}$} \\
\hline & & (ii) & Ordered GP [2] zones & \\
\hline & & (iii) & $\theta^{\prime \prime}$ phase & \\
\hline & & (iv) & $\theta^{\prime}$ phase & \\
\hline \multirow[t]{2}{*}{$\mathrm{Al}$} & $\mathrm{Mg}, \mathrm{Si}$ & (i) & $\begin{array}{l}\text { GP zones rich in } \mathrm{Mg} \\
\text { and } \mathrm{Si} \text { atoms }\end{array}$ & \multirow[t]{2}{*}{$\beta^{\prime \prime}-\mathrm{Mg}_{2} \mathrm{Si}$} \\
\hline & & (ii) & Ordered zones of $\beta^{\prime \prime}$ & \\
\hline \multirow[t]{2}{*}{$\mathrm{Al}$} & $\mathrm{Mg}, \mathrm{Cu}$ & (i) & $\begin{array}{l}\mathrm{GP} \text { zones rich in } \mathrm{Mg} \\
\text { and } \mathrm{Cu} \text { atoms }\end{array}$ & \multirow[t]{2}{*}{$S-\mathrm{CuAl}_{2} \mathrm{Mg}$} \\
\hline & & (ii) & $S^{\prime}$ platelets & \\
\hline \multirow[t]{2}{*}{$\mathrm{Al}$} & $\mathrm{Mg}, \mathrm{Zn}$ & (i) & $\begin{array}{l}\text { Spherical zones rich in } \\
\mathrm{Mg} \text { and } \mathrm{Zn}\end{array}$ & \multirow{2}{*}{$\eta-\mathrm{MgZn}_{2}$} \\
\hline & & (ii) & Platelets of $\eta^{\prime}$ phase & \\
\hline
\end{tabular}

Of the three steps, quenching has the most lasting effects on the material, as a slow quench will limit the hardenability, and a fast quench will give the best mechanical properties. To this end, Jominy and Boegehold created a test for the hardenability of steel that was eventually adopted by ASTM as the ASTM A255 standard $[14,15]$. The test involved heating a bar of steel to the austenitizing temperature for an hour, then placing it in a holder above a stream of water. After the bar has cooled to room temperature, a flat is ground off of it and hardness indents are done along the length of the bar. This testing method was adopted for aluminum alloys, with Newkirk and MacKenzie summarizing much of the test developmental work [14]. The Jominy End Quench (JEQ) test is attractive for aluminum alloys as it gives many quench rates without the need for creating multiple samples of one alloy [14].

Precedent has been set in literature, specifically by Ma et al [16], in testing other aluminum alloys using the JEQ test. In the Ma study, they cast A356 in permanent molds, placed thermocouples along the length of the bar, quenched the bars, and then took hardness data from along the length of the bars. Figure 2-1 illustrates the quench sensitive nature of A356, though it is not as sensitive as an alloy from the 7000 series aluminum alloys. 


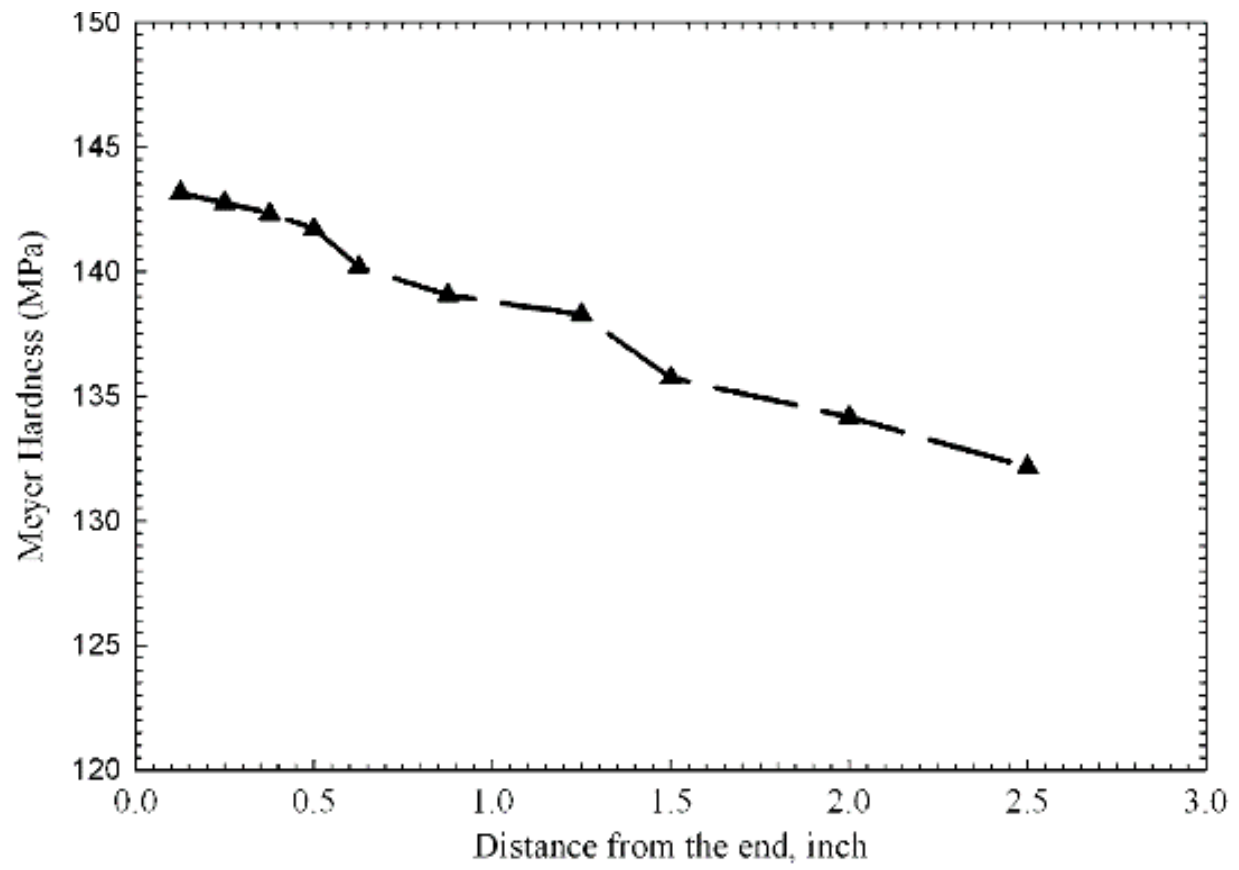

Figure 2-1: Meyer hardness along a Jominy end quench bar of cast aluminum A356 [16].

This need for a fast quench is best shown within the 7000 series aluminum alloys, as the kinetics for the $\eta$ phase formation are very fast. Because of this, 7000 series aluminum alloys are said to be quench sensitive, as they have a high degree of difficulty in terms of cooling the alloy quickly enough throughout the sample to keep the strengthening phase in solid solution [7]. However, there is a difference between the quench sensitivity of alloys within the same family, which can be seen when comparing between two 7000 series alloys, specifically 7050 and 7075 . The quench sensitivity of the two of them varies greatly as shown by Newkirk et al. in Figure 2-2. Of note is the difference between the amounts of chromium and zirconium. The 7075 used in the study had 0.20 weight $\%$ chromium and 0.0115 weight $\%$ zirconium. This is in comparison to the 7050 having 0.026 weight $\%$ chromium and 0.09 weight $\%$ zirconium [14]. Wagner et al. postulated that zirconium additions may reduce quench sensitivity, as the $\mathrm{Al}_{3} \mathrm{Zr}$ particles do not act as nucleation sites for GP zones. However, in the presence of chromium additions, the quench sensitivity increases, as chromium wants to precipitate out during a slow quench and act as nucleation sites for strengthening phases, with the ideal being low chromium and some zirconium present in the alloy [17]. 


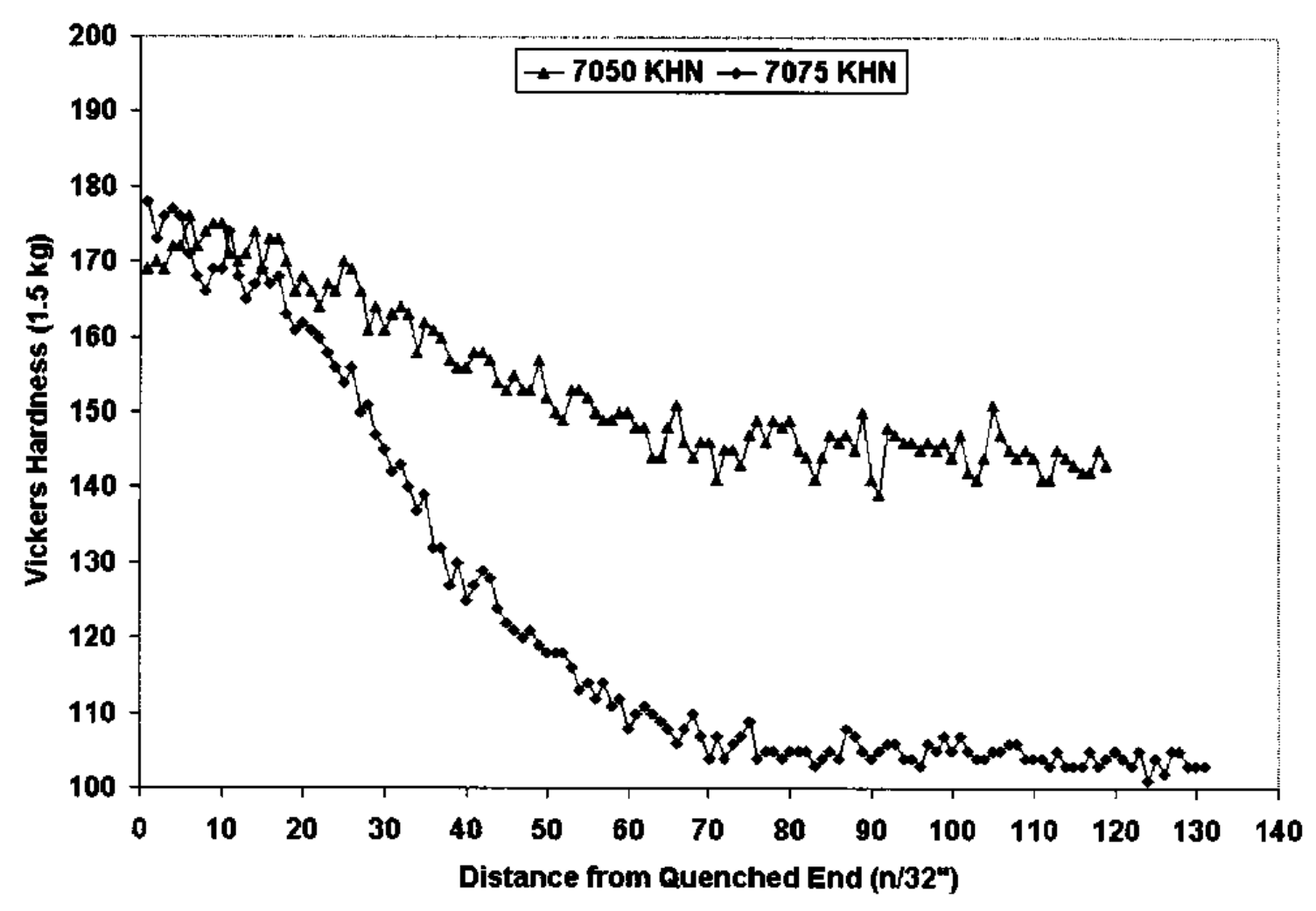

Figure 2-2: Hardness profile of 7075 and 7050 aluminum Jominy end quench specimens [14].

It has been shown that testing the end quenched samples using both hardness and electrical conductivity give insight into the effectiveness of the quench [7]. Electrical conductivity of aluminum alloys is related to the level of the solute in the matrix and the distortion energy between the precipitate phases in solid solution, and once the solute is out of solution, the effect on conductivity is reduced $[7,18,19]$. This is why there is low conductivity at the quenched end and increasing conductivity along the length of the bar, because the amount of solute in the matrix is changing with quench rate.

\section{Experimental Methods}

\subsection{Solidification Trials}

\subsubsection{Casting}

The experiments for this work were all based on the JEQ and multiple solidification rates. The solidification rates that were seen in the complex casting were; a slow cooling rate near the bottom of the mold by the reservoir of metal, a faster cooling rate in the middle of the mold, and a very fast cooling rate at the top of the mold by the chill, so a sand mold, a chilled sand mold and a permanent mold were used to simplify the solidification rates. The samples made were a variation on the design of the JEQ bar, 
which is usually a cylinder of $100 \mathrm{~mm}$ (3.94 inches) in length and $25 \mathrm{~mm}(0.98425$ inches) in diameter. The bars cast were six inches long with a one inch diameter, with a flat designed into that bar that was 0.1 inches $(2.54 \mathrm{~mm})$ deep, that was offset from the end of the bar by one inch. The JEQ bars themselves were cut off of the casting at the junction of the runner and the bar (Figure 3-1). This flat was added to make the machining after quenching more efficient. Thermocouples were to be used to measure temperature data on the complex casting and relate it to the JEQ castings, however, due to the casting process for the complex casting, the data was not useful.

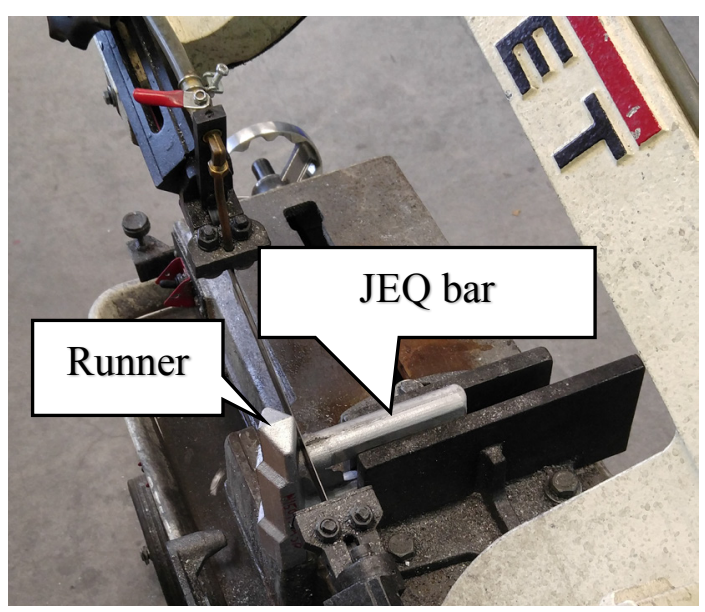

Figure 3-1: JEQ bar being cut off of the runner before being sent to get machined to proper length

Casting trials were done using three molds:

- A sand molded with a 3D printed pattern, whose initial surface finish was sanded down

- A sand mold that in addition to the 3D printed pattern also used grey iron chills

- A grey iron permanent mold

The sand molds were made using the 3D printed part in a sand mold box, where the 3D printed part was attached to a base plate, and walls were constructed around the base plate (Figure 3-2 \& Figure 3-3). 


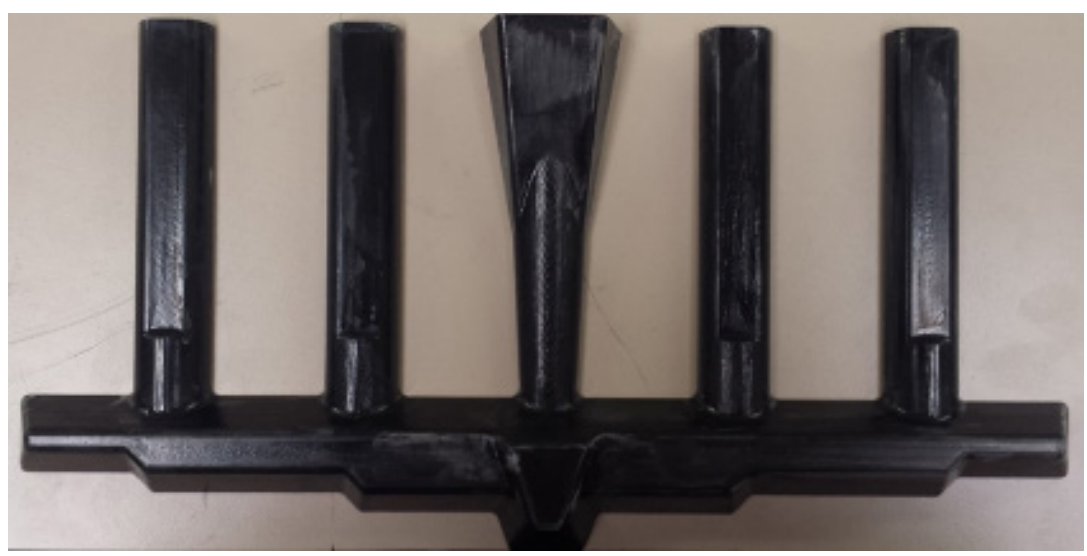

Figure 3-2: First iteration of 3D printed JEQ bar casting that was used to create sand mold box.

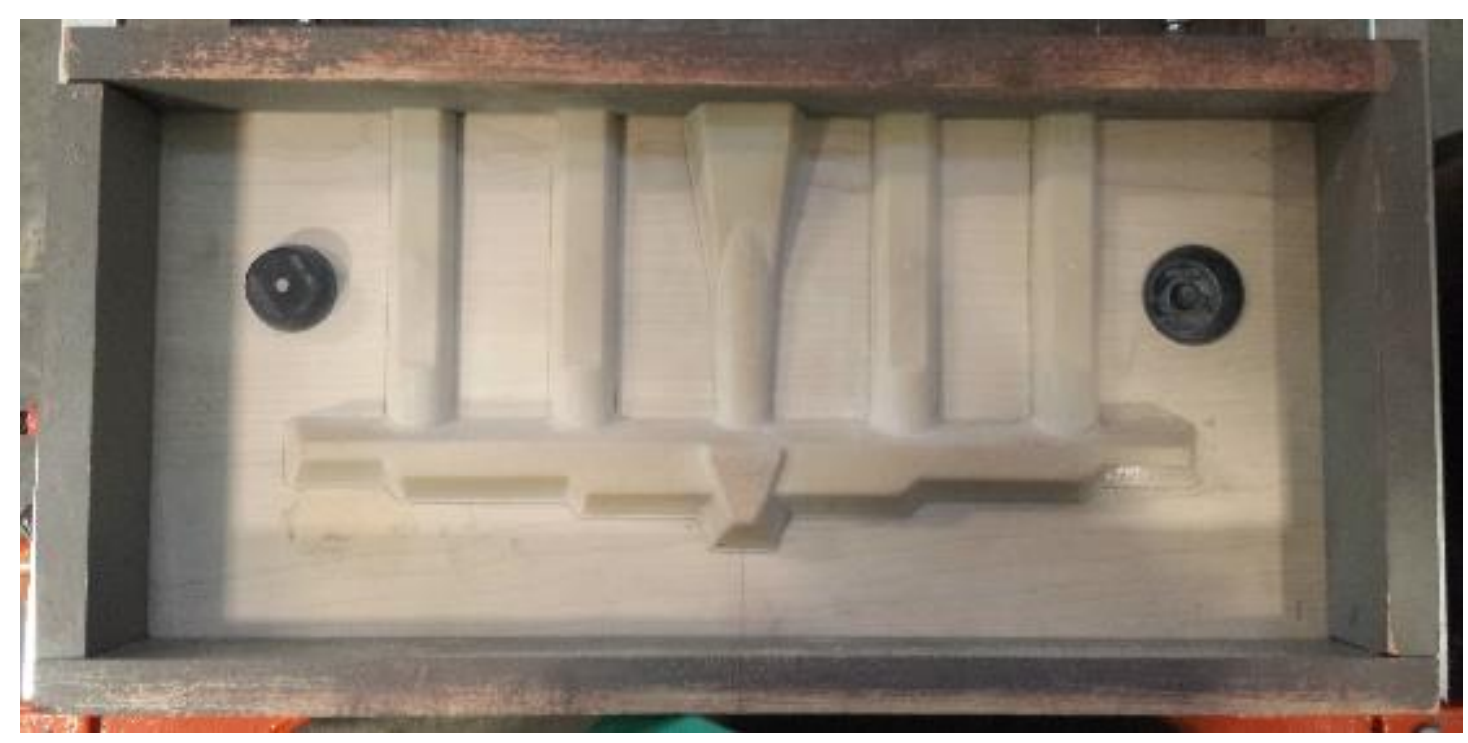

Figure 3-3: Finalized sand mold box, with second iteration 3D printed JEQ bar casting, also showing location features attached to the base plate.

The three molds were then clamped together in preparation for casting (Figure 3-4). A sample was also cast for optical emission spectroscopy. 


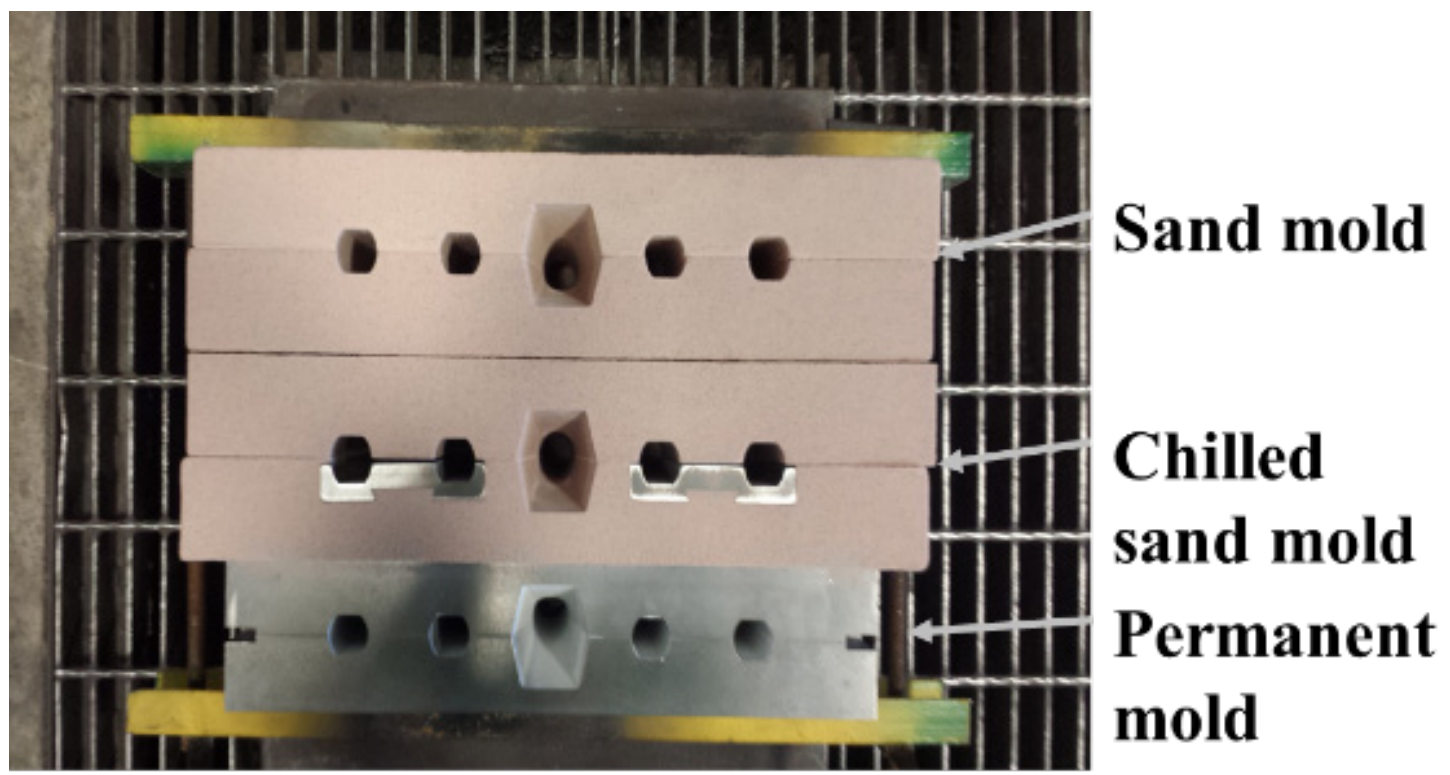

Figure 3-4: Molds ready for casting, clamped into place, with the sand, chilled sand, and permanent mold aligned properly. Note grey iron chills in chilled sand mold

The following casting parameters were recorded:

- The pour weights for each alloy

- The holding temperatures

- The pour temperatures for each alloy (Table 3-1)

The differences in pour temperatures relate to each individual alloy having a different melting temperature, while the differences in pour weights is an artifact of the charge material shape. All the alloys cast came from ingots that had to be cut into a shape and size that would fit into the furnace, as such the weights are different.

The nominal compositions compared to the actual casting composition (Table 3-2). The key elements to focus on are the chromium and zirconium levels, as well as the iron content. The castings were done using an induction furnace, with a cover gas, to limit the amount of oxide formation on the surface of the liquid metal. The melts were also degassed using argon and a graphite rotor, each melt was degassed for approximately ten minutes. 
Table 3-1: Casting Parameters

\begin{tabular}{|c|c|c|c|}
\hline Alloy & Pour Weight & $\begin{array}{c}\text { Holding } \\
\text { Temperature }\end{array}$ & Pour Temperature \\
\hline $\mathbf{A 2 0 6}$ & 14.1 lbs. & $750^{\circ} \mathrm{C}$ & $800^{\circ} \mathrm{C}$ \\
\hline $\mathbf{7 0 7 5}$ & 20.55 lbs. & $735^{\circ} \mathrm{C}$ & $740^{\circ} \mathrm{C}$ \\
\hline $\mathbf{3 1 9}$ & 17.30 lbs. & $704^{\circ} \mathrm{C}$ & $710^{\circ} \mathrm{C}$ \\
\hline $\mathbf{3 5 5}$ & 16.70 lbs. & $710^{\circ} \mathrm{C}$ & $715^{\circ} \mathrm{C}$ \\
\hline $\mathbf{A 3 5 6}$ & 18.85 lbs. & $712.8^{\circ} \mathrm{C}$ & $720^{\circ} \mathrm{C}$ \\
\hline
\end{tabular}

Table 3-2: Nominal and Actual compositions used for cast alloys [4]

\begin{tabular}{|c|c|c|c|c|c|c|c|c|c|c|c|c|c|c|c|c|c|}
\hline & \multicolumn{9}{|c|}{ Nominal Compositions (wt \%) } & \multicolumn{6}{|c|}{ Actual Compositions (wt \%) } \\
\hline Alloys & $\mathbf{C u}$ & $\mathbf{S i}$ & $\mathbf{M n}$ & $\mathbf{M g}$ & $\mathbf{T i}$ & $\mathbf{Z n}$ & $\mathbf{C r}$ & $\mathbf{F e}$ & $\mathbf{C u}$ & $\mathbf{S i}$ & $\mathbf{M n}$ & $\mathbf{M g}$ & $\mathbf{T i}$ & $\mathbf{Z n}$ & $\mathbf{Z r}$ & $\mathbf{C r}$ & $\mathbf{F e}$ \\
\hline $\mathbf{A 2 0 6}$ & 4.6 & - & 0.35 & 0.25 & 0.22 & - & - & - & 4.6 & 0.05 & 0.27 & 0.27 & 0.21 & 0.22 & 0.08 & $<0.01$ & 0.05 \\
\hline $\mathbf{7 0 7 5}$ & 1.6 & - & - & 2.5 & 0.23 & 5.6 & - & - & 1.9 & 0.07 & 0.04 & 2.3 & $<0.01$ & 5.9 & 0.07 & 0.29 & 0.3 \\
\hline $\mathbf{3 1 9}$ & 3.5 & 6 & - & - & - & - & - & - & 3.6 & 7.2 & 0.02 & 0.18 & 0.14 & 0.03 & 0.13 & $<0.01$ & 0.12 \\
\hline $\mathbf{3 5 5}$ & 1.3 & 5 & - & 0.5 & - & - & 0.25 & - & 1.7 & 8.9 & $<0.01$ & 0.33 & 0.06 & 0.01 & 0.08 & $<0.01$ & 0.14 \\
\hline $\mathbf{A 3 5 6}$ & - & 7 & - & 0.35 & - & - & - & - & $<0.01$ & 7.3 & $<0.01$ & 0.38 & 0.13 & 0.02 & 0.08 & $<0.01$ & 0.12 \\
\hline
\end{tabular}

\subsubsection{Secondary Dendrite Arms Spacing (SDAS)}

Before the JEQ test was done to quantify the quench sensitivity of the samples, more characterization experiments had to be conducted to quantify the solidification rate of the alloys. To this end, representative samples were cut off of other bars, about 0.5 inches $(12.7 \mathrm{~mm})$ from the top of the casting. These samples were mounted in epoxy, ground, polished and etched with Keller's reagent. They were then imaged with the express purpose of being used for SDAS measurements. The process for doing these measurements is:

- $\quad$ Take 10 images of a sample at differing objective magnifications as the sample dictated

- $\quad$ Set scale for each image in ImageJ, by using an image of a micrometer with each different objective, and calibrating the set distance of the micrometer to the number of pixels measured

- $\quad$ Measure dendrite spacing using the calibrated image in ImageJ, by measuring a line from edge to edge of the dendrites and then dividing by the number of dendrites (Figure 3-5) 


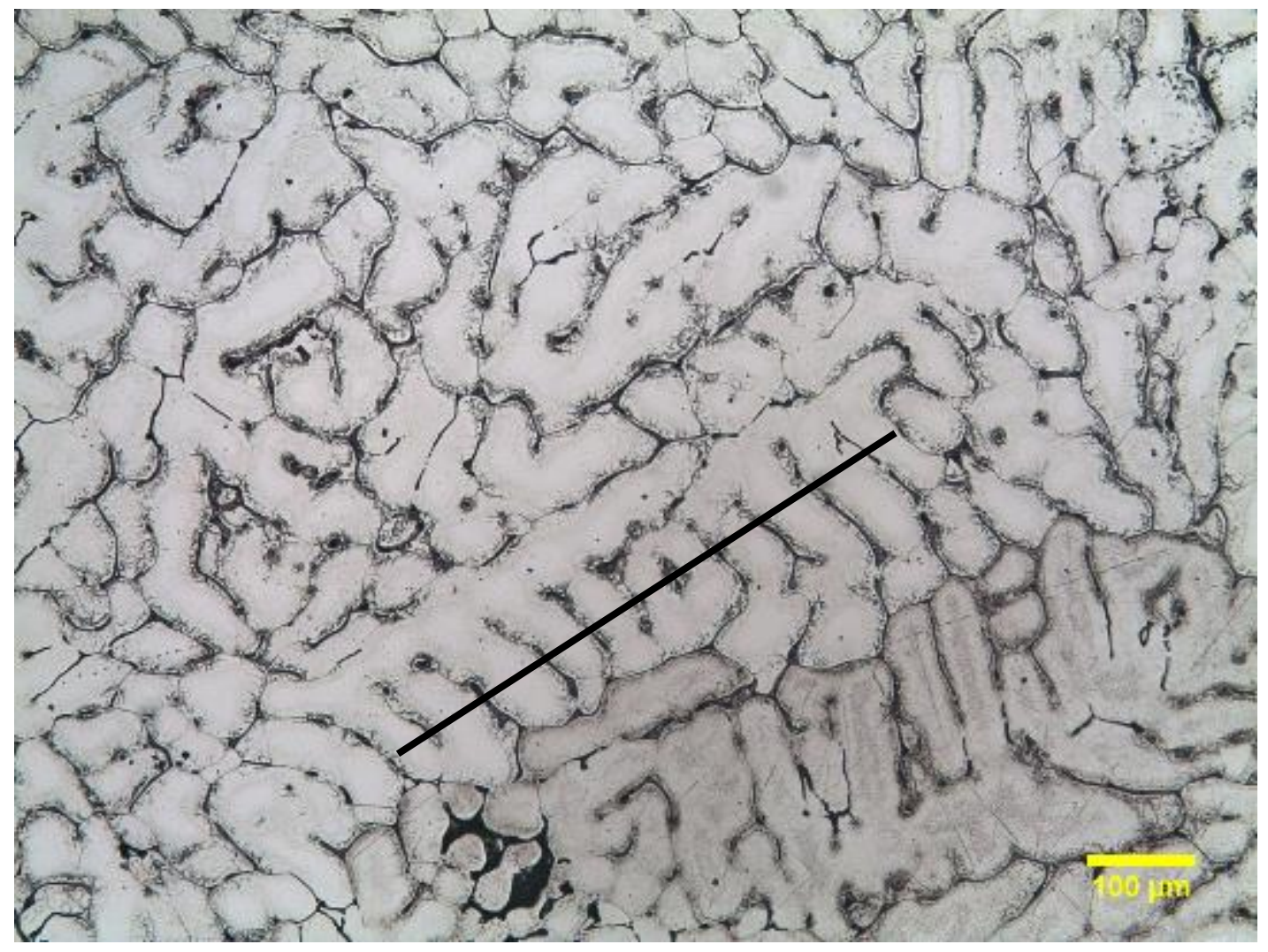

Figure 3-5: Sample of SDAS measurement image, with scale bar and line measuring distance from one dendrite to another, image is of 7075 cast in sand.

\subsubsection{Porosity Measurement}

To measure the soundness of the casting, porosity measurements were done using the same samples from the SDAS measurements. The porosity measurements were done using the Archimedes' method with the process being:

- Weigh the samples dry, the dry weight

- Submerge samples in water and reweigh while submerged, the wet weight

The two weights were then used to calculate the density of the sample and then compared against the density of the alloy, referenced from literature [20]. Unfortunately, no corrections were done for water temperature or composition issues within the alloys, as the density was calculated automatically on a QCD-1 Specific Gravity and Porosity Measurement System. The Archimedes' method relates the mass of the object to the mass of the water it displaces and uses those two masses to calculate the density of the sample.

\subsubsection{Tensile Testing}

Other bars from the castings were solutionized, quenched, heat treated and machined into tensile bars. These bars were cut off of the casting and machined to the ASTM E8 
standard [21] and tested in the MTS 4206 at a strain rate of 10-3/s. An Epsilon axial extensometer was used in the gage length. The samples were all pulled to failure and the engineering stress-strain data were collected and analyzed.

\subsection{Quenching Trials}

\subsubsection{Jominy End Quench Test}

Bars were cut off of the casting, at the runner, and machined to the standard JEQ bar length [15] and tapped to stay in place in the JEQ testing apparatus. Four holes were drilled into the bar and thermocouples were placed along the length at $0.5,1.5,2.5$, and 3.5 inches $(12.7,38.1,63.5$, and $88.9 \mathrm{~mm})$ from the quenched end. The bars were then placed in a box furnace, on insulating blocks, set to the specific alloys' solutionizing temperature and solutionized following the standard procedure for each alloy (Table 3-3). The two step solutionizing that some of the alloys have is done to dissolve two different sets of precipitates in the alloy.

Table 3-3: Solutionizing Times and Temperatures

\begin{tabular}{|c|c|c|}
\hline Alloy & $\begin{array}{c}\text { Solutionizing } \\
\text { Temperature }\end{array}$ & Solutionizing Time \\
\hline $\mathbf{A 2 0 6}$ & $510-515.5^{\circ} \mathrm{C}, 526.6-$ & 2 hrs., $14-20 \mathrm{hrs}$. \\
\hline $\mathbf{7 0 7 5}$ & $465.2^{\circ} \mathrm{C}$ & 4 hrs. \\
\hline $\mathbf{3 1 9}$ & $501.6-507.2^{\circ} \mathrm{C}$ & $12 \mathrm{hrs}$. \\
\hline $\mathbf{3 5 5}$ & $504.4^{\circ} \mathrm{C}, 535^{\circ} \mathrm{C}$ & $2 \mathrm{hrs} ., 6 \mathrm{hrs}$. \\
\hline $\mathbf{A 3 5 6}$ & $540.5^{\circ} \mathrm{C}$ & $12 \mathrm{hrs}$. \\
\hline \multicolumn{2}{|c}{} \\
\hline
\end{tabular}

The procedure for running the JEQ test followed a set of steps:

- Turn on data acquisition unit to begin getting temperature data from the bar, using $\mathrm{K}$ type thermocouples at a rate of approximately $2 \mathrm{~Hz}$

- Open furnace and retrieve bar with tongs, retrieval time of approximately 2-5 seconds

- Place bar in JEQ apparatus and turn on water

- Turn off water once all thermocouples read approximately $32^{\circ} \mathrm{C}$

Figure 3-6 shows the JEQ test in action. Note the four thermocouple locations, as well as the insulating ceramic disk used to thermally isolate the metallic washer (and JEQ bar) from contacting the apparatus, as the metal of the apparatus would act as a chill and cause cooling from both ends of the bar. 


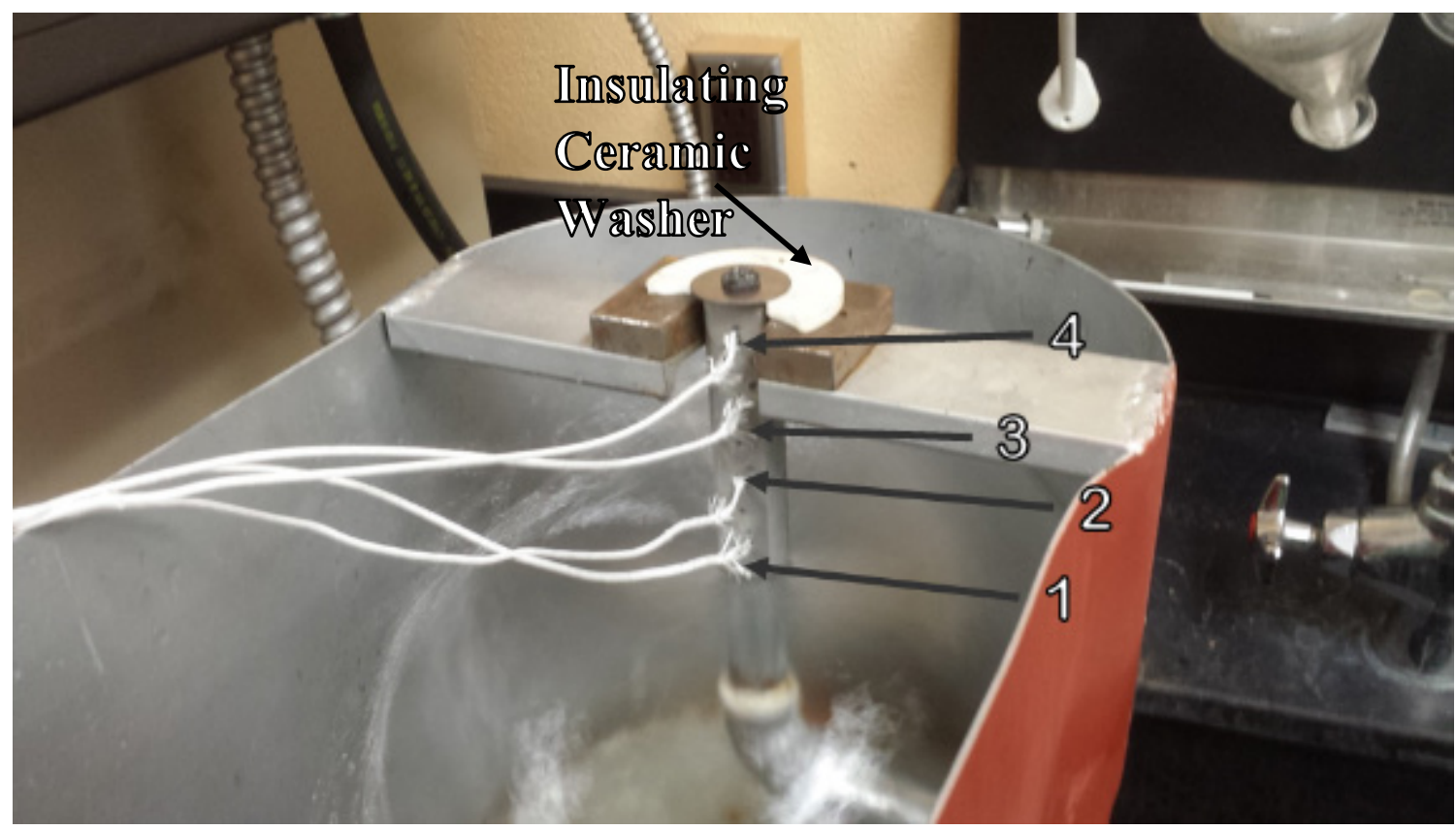

Figure 3-6: JEQ test in operation, with thermocouples at 0.5, 1.5, 2.5, and 3.5 inches $(12.7,38.1,63.5$, and $88.9 \mathrm{~mm})$ along the length of the bar, corresponding to labels 1 through 4, respectively. The ceramic washer is 0.146 inches $(3.7084 \mathrm{~mm})$ thick.

The bars were then heat treated to T6, the peak aged temper, with temperatures and times given (Table 3-4). The T6 temper was selected to compare all the alloys on the same temper, despite that in applications, the tempers would be very different for each alloy.

Table 3-4: T6 Heat Treating Temperatures and Times

\begin{tabular}{|c|c|c|}
\hline Alloy & $\begin{array}{c}\text { Heat Treating } \\
\text { Temperature }\end{array}$ & Heat Treating Time \\
\hline $\mathbf{A 2 0 6}$ & $151.6-157.2^{\circ} \mathrm{C}$ & $20 \mathrm{hrs}$. \\
\hline $\mathbf{7 0 7 5}$ & $121.1^{\circ} \mathrm{C}$ & $24 \mathrm{hrs}$. \\
\hline $\mathbf{3 1 9}$ & $151.6-157.2^{\circ} \mathrm{C}$ & $2-5 \mathrm{hrs}$. \\
\hline $\mathbf{3 5 5}$ & Room Temperature, & Proprietary \\
\hline $\mathbf{A 3 5 6}$ & $154.4^{\circ} \mathrm{C}$ & $2-5 \mathrm{hrs}$. \\
\hline
\end{tabular}

\subsubsection{Hardness Testing}

The heat treated bars were then milled down 0.1 inches $(2.54 \mathrm{~mm})$ on both sides, and one side was ground and polished in preparation for hardness testing (Figure 3-7). 


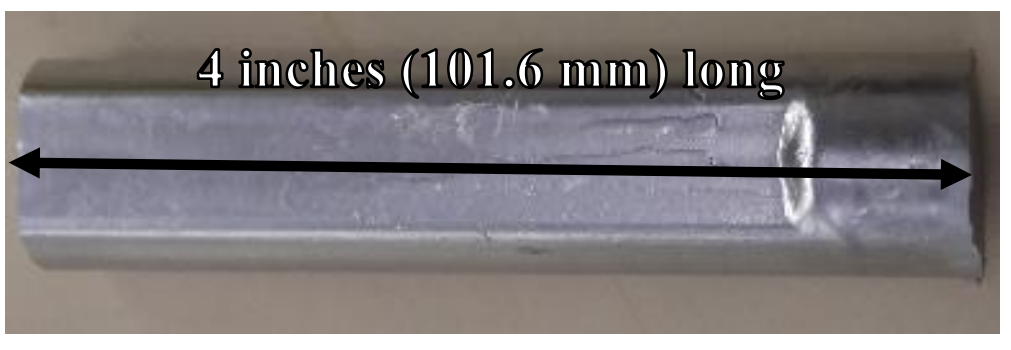

Figure 3-7: Cast JEQ bar, pre-machining and before any testing has taken place

Hardness testing was done on a LECO MHT 200, with the JEQ bars using a custom mounting platform to have repeatable indents done on the samples, as the normal sample holder was not stable enough. The testing parameters for the JEQ bars using the Vickers indenter were: a 15 second dwell time, a 500 gram load, and the 20 times objective. In the case of the JEQ bar, the hardness indents were done at $1 / 8^{\text {th }}$ inch $(3.175 \mathrm{~mm})$ intervals, in groups of three (Figure 3-8).

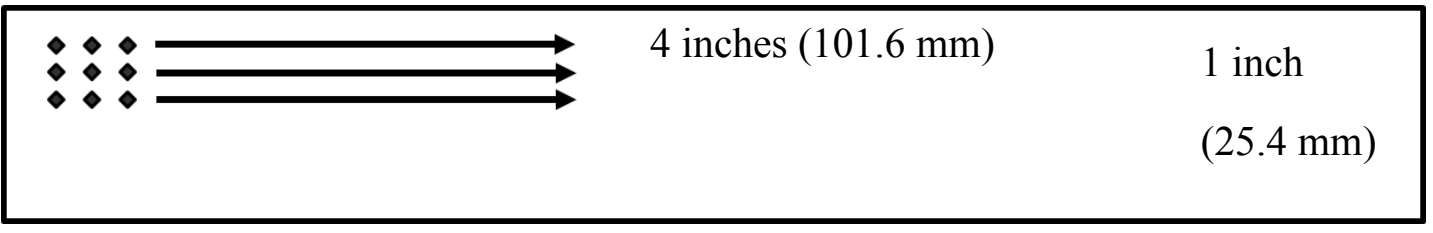

Figure 3-8: Schematic of hardness testing, with indents every $3.175 \mathrm{~mm}$ in the $x$-direction and every $2 \mathrm{~mm}$ in the $y$-direction.

\subsubsection{Electrical Conductivity}

Another measure of how quench sensitive an alloy can be is to measure the electrical conductivity of the sample after the end quench. There is a direct correlation between conductivity and percentage of the strengthening phase in solid solution. Measuring the conductivity was done with a Fischer SigmaScope SMP10 probe with a diameter of 6 $\mathrm{mm}$ along the length of the bar at quarter inch $(6.35 \mathrm{~mm})$ intervals, in groups of three. The conductivity was measured after each sample was re-solutionized and end quenched, with each bar then being placed in a freezer at $-80^{\circ} \mathrm{C}$. This was done to keep natural aging from taking place, as natural aging would grow precipitates and mitigate the differences of the quench effectiveness. 


\section{Results}

\subsection{Solidification Trials}

\subsubsection{Secondary Dendrite Arms Spacing}

SDAS measurements were averaged over the whole of the sample (Table 8-1, Figure 4-1). In the 319 sand cast sample there were not enough dendrites for statistical analysis, as the dendrites had grown in a direction that was not easily visible from the sample surface. More examples of the micrographs used for SDAS measurements are given in Appendix B: Selected SDAS images for all alloys, specifically showing trends in the alloys as the solidification rate changes across the mold types used.

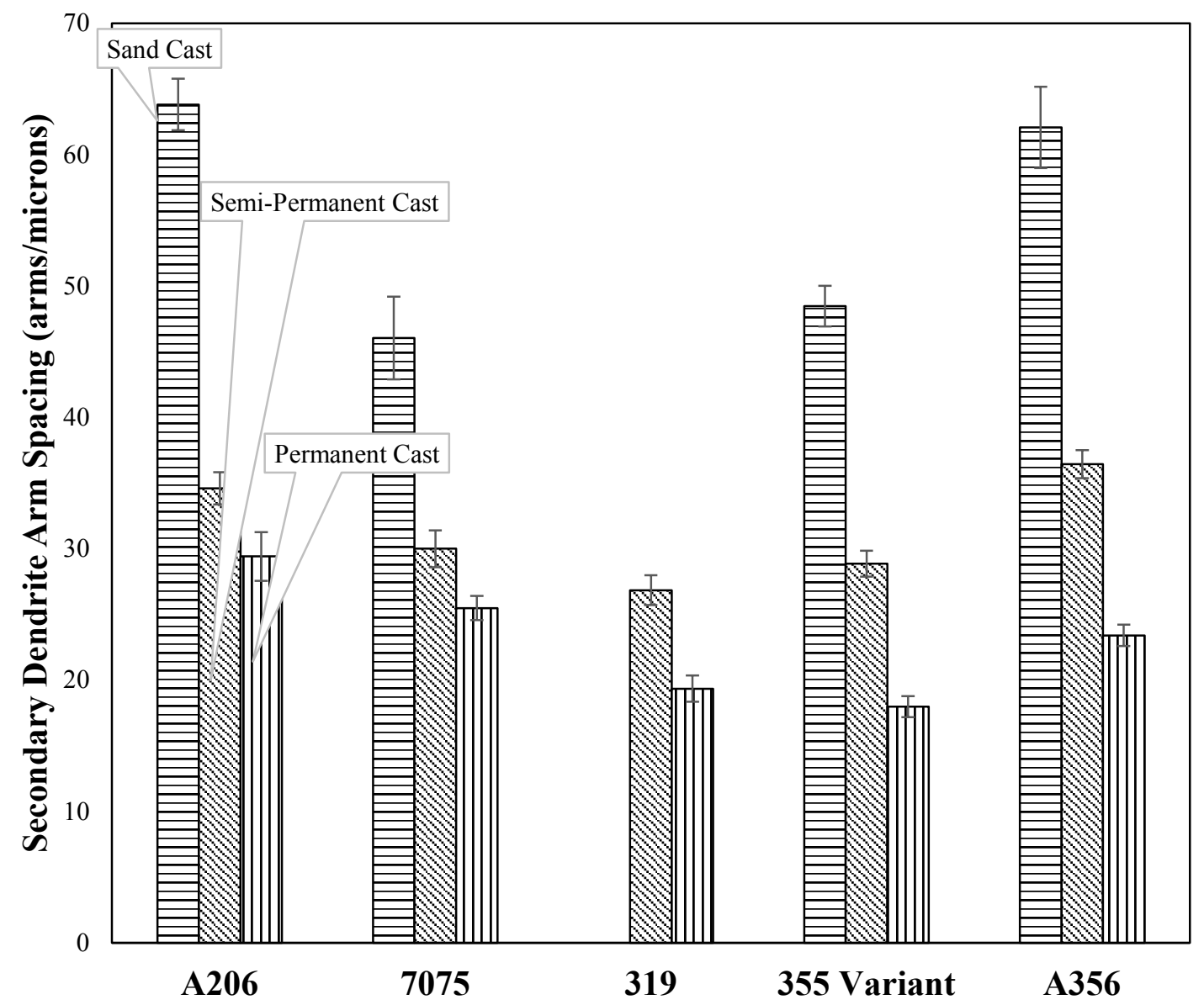

Figure 4-1: Experimental SDAS averages from all the alloy/mold combinations with 95\% confidence shown.

\subsubsection{Porosity Measurements}

Porosity data from the measurements were collected (Table 8-2, Figure 4-2). The porosity data, specifically the amount of porosity, will correlate inversely to the 
elongation achievable (Figure 4-3). Two of the observed trends shown by the data do not follow any expected trends, as the porosity decreases for 319 and A356 as solidification rate decreases. The spike in 355 at the semi-permanent cast sample does not follow either trend. The trends seen in A206 and 7075, however do follow expected trends, with the permanent cast 7075 sample showing the worst $\%$ porosity.

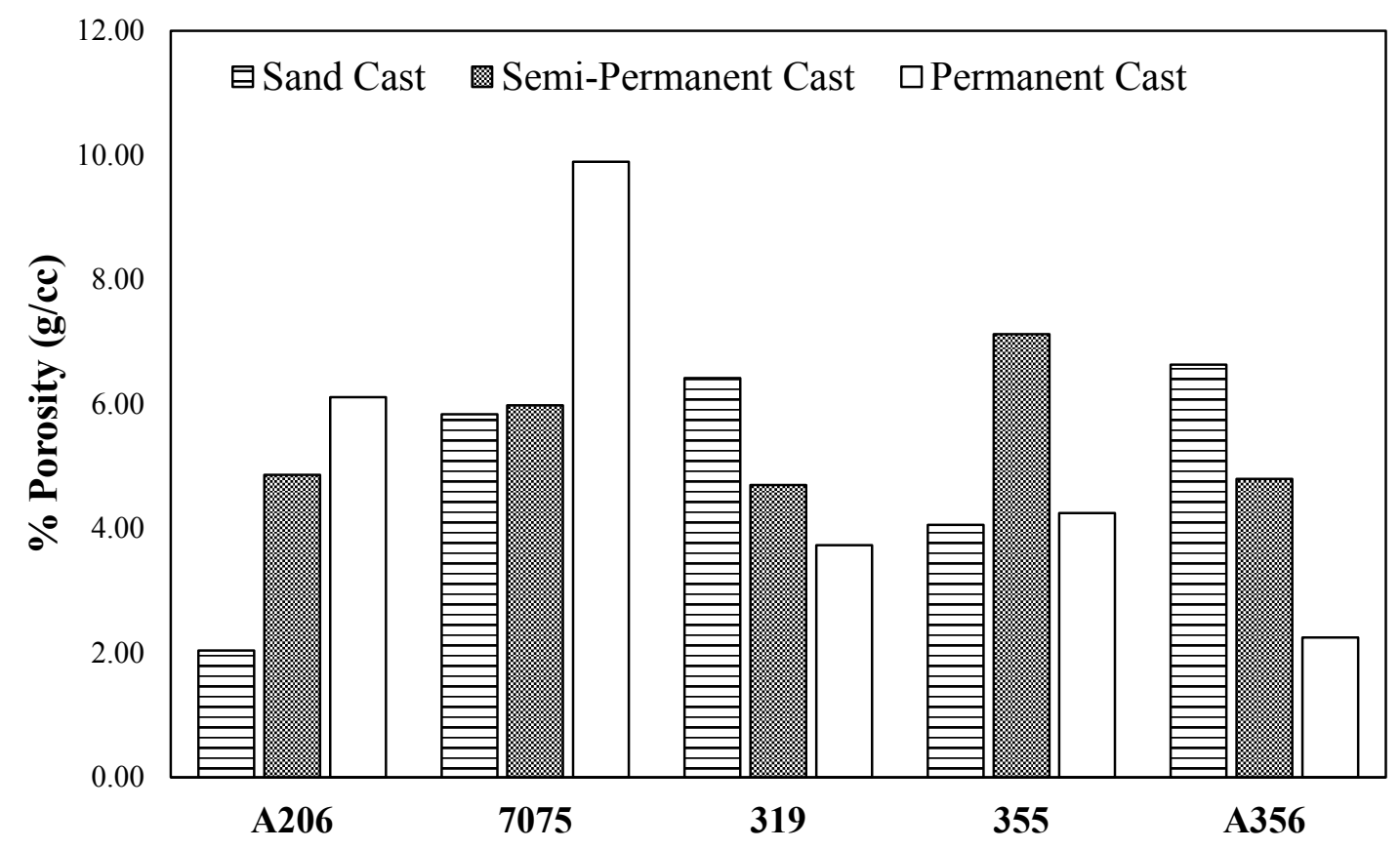

Figure 4-2: Porosity as of percentage difference between calculated density and referenced density from literature [20].

\subsubsection{Tensile Testing}

Some tensile bars from the castings had shrinkage in the top of the bar, which adversely affected the gripping ability of the tensile tester. Specifically the permanent mold cast A206 and the permanent mold cast 355 had issues where they had to be pulled again. The data was collated into a simple to read format (Table 4-1). An example of the tensile tests in the familiar stress-strain form is given in Figure 4-3. 355 follows the trend expected, that as solidification rate decreases, the tensile strength increases. The rest are plagued by porosity issues that reduce the achievable tensile strength. Most of the samples did not break in the gage length, but broke where the extensometer made contact with the gage length. 
Table 4-1: Elastic Modulus, Yield Stress, Ultimate Stress, and Strain for Alloys cast

\begin{tabular}{|c|c|c|c|c|c|}
\hline Alloy & Mold & $\begin{array}{l}\text { Modulus } \\
\text { (GPa) }\end{array}$ & $\begin{array}{l}\text { Yield Stress } \\
\text { (MPa) }\end{array}$ & $\begin{array}{c}\text { Ultimate } \\
\text { Stress (MPa) }\end{array}$ & $\begin{array}{c}\text { Strain at } \\
\text { break }\end{array}$ \\
\hline \multirow{3}{*}{ A206 } & Sand & 67.7 & 248 & 371 & 0.09 \\
\hline & $\begin{array}{c}\text { Semi- } \\
\text { Permanent }\end{array}$ & 61.6 & 237 & 357 & 0.088 \\
\hline & Permanent & 56.5 & 234 & 367 & 0.104 \\
\hline \multirow{3}{*}{7075} & Sand & 51.8 & 105 & 109 & 0.005 \\
\hline & $\begin{array}{c}\text { Semi- } \\
\text { Permanent }\end{array}$ & 57.8 & 119 & 182 & 0.02 \\
\hline & Permanent & 51.4 & 119 & 157 & 0.013 \\
\hline \multirow{3}{*}{319} & Sand & 73.8 & 203 & 265 & 0.014 \\
\hline & $\begin{array}{c}\text { Semi- } \\
\text { Permanent }\end{array}$ & 75.3 & 216 & 329 & 0.033 \\
\hline & Permanent & 65.9 & 213 & 331 & 0.037 \\
\hline \multirow{3}{*}{355} & Sand & 80.6 & 217 & 251 & 0.01 \\
\hline & $\begin{array}{c}\text { Semi- } \\
\text { Permanent }\end{array}$ & 71.4 & 236 & 332 & 0.049 \\
\hline & Permanent & 66.2 & 315 & 325 & 0.02 \\
\hline \multirow{3}{*}{ A356 } & Sand & 79.5 & 182 & 218 & 0.012 \\
\hline & $\begin{array}{c}\text { Semi- } \\
\text { Permanent }\end{array}$ & \multicolumn{4}{|c|}{ N/A } \\
\hline & Permanent & 62.8 & 196 & 293 & 0.066 \\
\hline
\end{tabular}




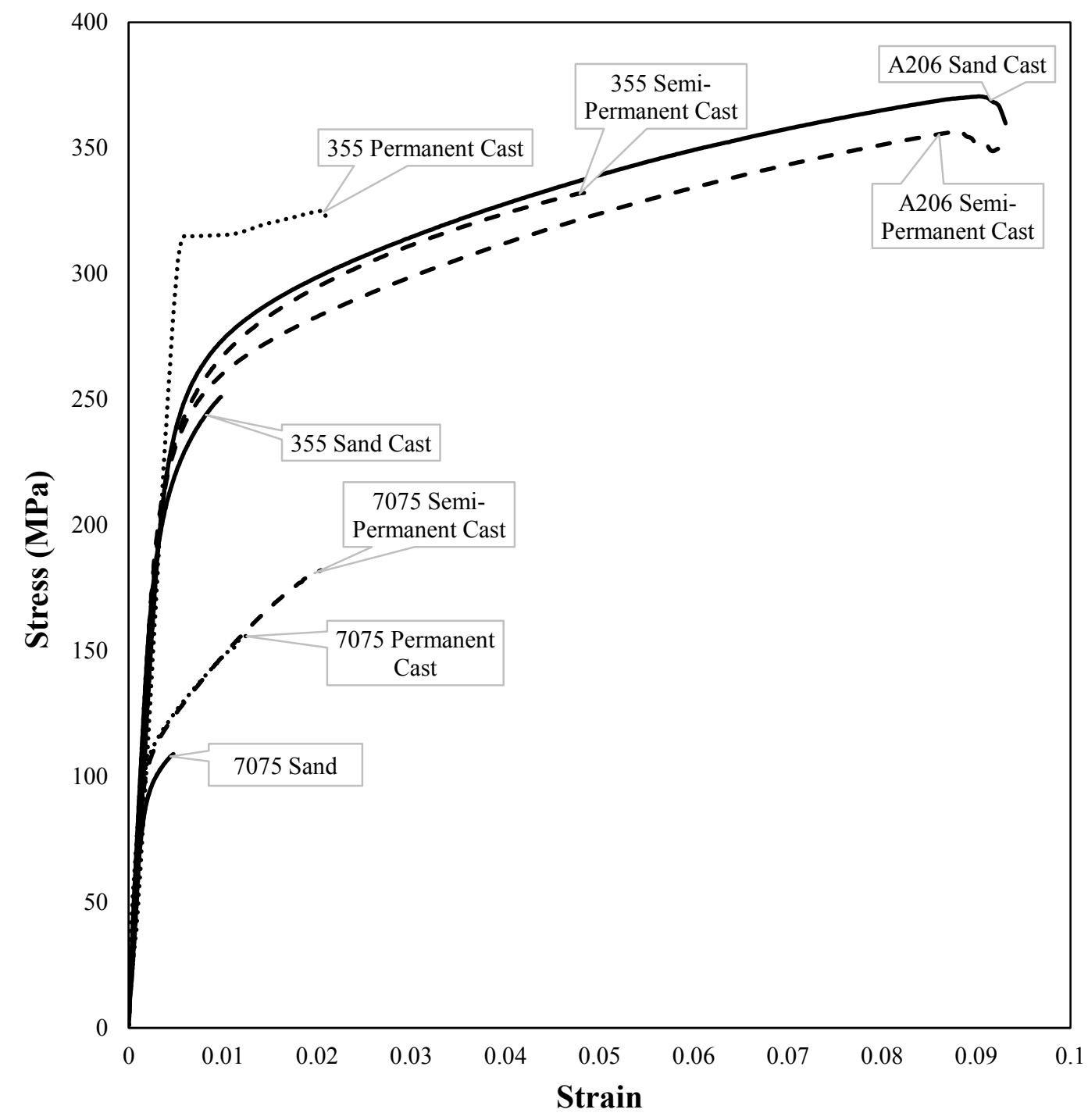

Figure 4-3: Stress-strain curves for A206, 7075, and 355 alloys, for all three mold types.

\subsection{Quenching Trials}

\subsubsection{Hardness Tests}

The data collected from the hardness tester was averaged (Figure 4-4). 7075 samples followed expected trends, while the other data had a parabolic nature. 


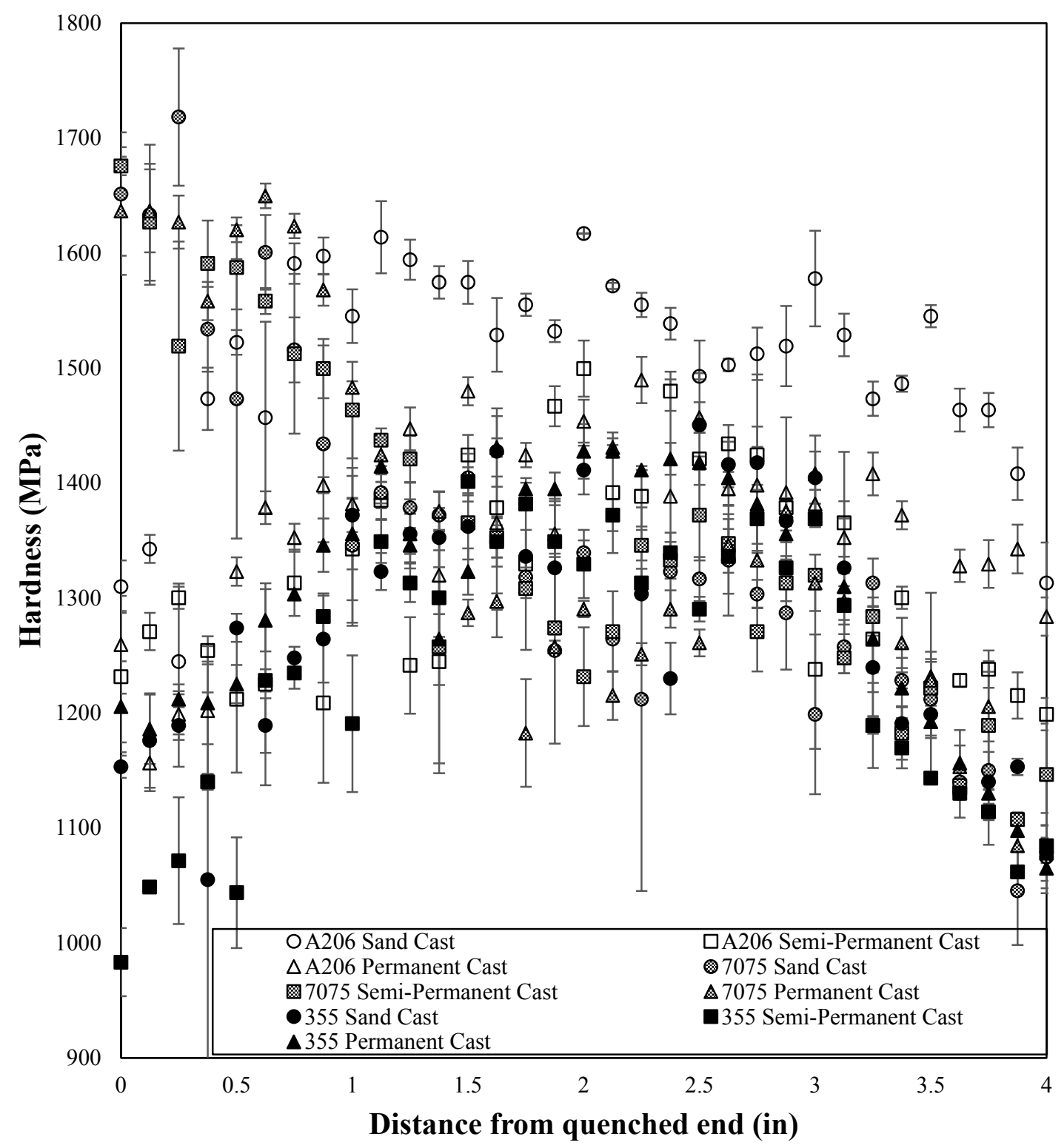

Figure 4-4: Hardness data down the length of the bar for A206, 7075 and 355 alloys in each mold type with 95\% confidence shown.

\subsubsection{Electrical Conductivity}

The electrical conductivity data was averaged over the groups of three, graphed against the distance from the quenched end. It shows how effective the quench was along the length of the bars, and might be able to help explain why there are issues with the hardness data (Figure 4-5). 7075 followed expected trends for a quench sensitive alloy, while the other alloys followed different trends. 


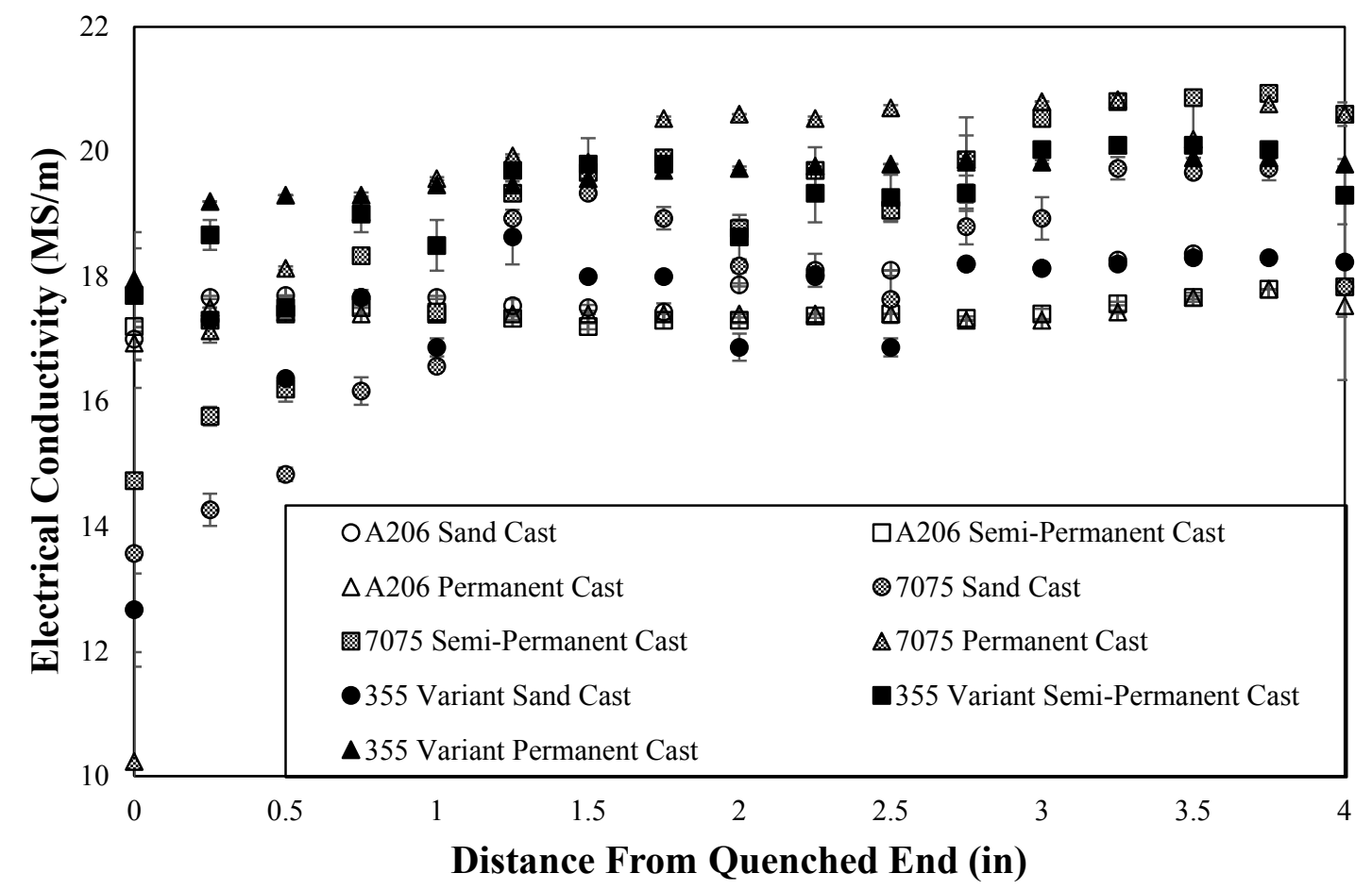

Figure 4-5: Electrical conductivity down the length of the bar for A206, 7075 and 355 alloys, in each mold type with $95 \%$ confidence shown.

\section{Discussions}

\subsection{Solidification Trials}

The solidification rates observed in the castings were calculated by using the SDAS and a general relation in Equation 1. However, there are other equations that are more specific for each alloy, using alloy specific coefficients.

$$
-\log (R)=-2.5 * \log \left(\lambda_{s}\right)+4.5[22]
$$

Table 5-1 summarizes the data from the calculations. An issue is that the data is calculated data, not actual experimentally collected data collected live from the thermocoupled molds as the metal was cooling. Solidification rate comparison between the simple castings and the complex casting could be done using data from thermocoupled molds for the simple casting and thermocouple data from the complex casting. 
Table 5-1: Calculated solidification rates for alloys cast

\begin{tabular}{|c|c|c|}
\hline Alloy & Mold Type & $\begin{array}{c}\text { Calculated } \\
\text { Solidification } \\
\text { Rate }\left({ }^{\circ} \mathbf{C} / \mathbf{s}\right)\end{array}$ \\
\hline \multirow{4}{*}{ A206 } & Sand & 0.97 \\
\cline { 2 - 3 } & $\begin{array}{c}\text { Semi- } \\
\text { Permanent }\end{array}$ & 4.5 \\
\cline { 2 - 3 } & Permanent & 6.7 \\
\hline \multirow{4}{*}{$\mathbf{7 0 7 5}$} & Sand & 2.2 \\
\cline { 2 - 3 } & $\begin{array}{c}\text { Semi- } \\
\text { Permanent }\end{array}$ & 6.4 \\
\cline { 2 - 3 } & Permanent & 9.6 \\
\hline \multirow{4}{*}{319} & Sand & N/A \\
\cline { 2 - 3 } & $\begin{array}{c}\text { Semi- } \\
\text { Permanent }\end{array}$ & 8.5 \\
\cline { 2 - 3 } & Permanent & 19 \\
\hline \multirow{4}{*}{355} & Sand & 1.9 \\
\cline { 2 - 3 } & $\begin{array}{c}\text { Semi- } \\
\text { Permanent }\end{array}$ & 7.1 \\
\cline { 2 - 3 } & Permanent & 23 \\
\hline \multirow{4}{*}{ 3356 } & Sand & 1.04 \\
\cline { 2 - 3 } & $\begin{array}{c}\text { Semi- } \\
\text { Permanent }\end{array}$ & 3.9 \\
\cline { 2 - 3 } & Permanent & 12 \\
\hline
\end{tabular}

The lack of good tensile strength, however, shows that something went wrong somewhere along the production of the bars, as some of the permanent cast bars showed obvious shrinkage cones in the top of the bars, with this cone correlating to the high amount of porosity seen in at least two of the alloys. Further investigations into the lack of ductility and tensile strength are needed.

Another issue is the amount of porosity. The porosity data partially follow trends from literature [1,2], where faster cooling rates are expected to have higher porosity. As shown in Figure 4-3 and Table 8-2, the amount of porosity correlates with the ductility of the samples, as many of the more porous samples have the lowest tensile strengths. This porosity is a mix of both gas porosity and inter-dendritic shrinkage porosity, with the shrinkage porosity being the dominant form of porosity. 


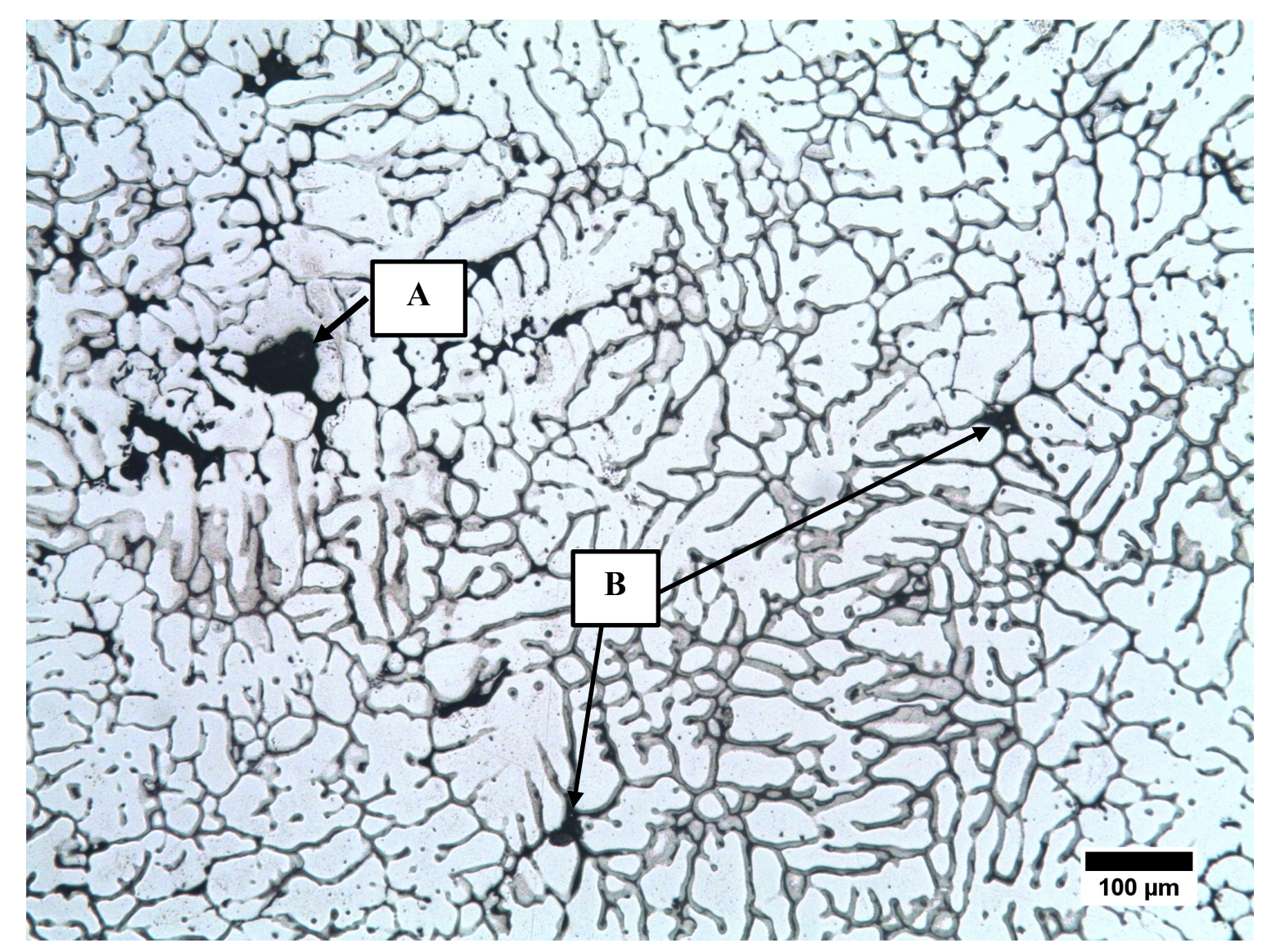

Figure 5-1: Micrograph of 7075 permanent cast, showing a) gas porosity and b) interdendritc shrinkage porosity

The difference between the two types of porosity can be seen in some of the micrographs given in Appendix B: Selected SDAS images for all alloys. The gas porosity was seen more heavily near the edges of the castings by where the metal came in contact with the mold walls. While degassing was done with all the castings, they were cast using an induction furnace. The induction fields from the furnace churned the liquid metal, exposing fresh metal to the surface, allowing for oxidation to happen. To mitigate this issue, a cover gas was used to limit this issue. A more in-depth look at the solidification modeling should clear up what type of porosity is the dominant form within the castings, and should help optimize the casting process. The modeling should focus on the solidification, specifically what phases will be present and what the porosity will be and where it will be located.

The tensile data followed trends dictated by the solidification structures. As the solidification rate increases, the yield strengths also increase, and this trend is seen in the 7075, the 355 and the A356. A206 behaves inversely, and 319 has its peak yield strength with the semi-permanent cast sample. However, when the tensile data is taken in 
conjunction with the porosity data, the influence of the porosity can be seen clearly, as the 7075 has lowest strength, both in terms of yield and ultimate strength (Figure 4-3).

\subsubsection{Quenching Trials}

Quench sensitivity relates directly to the strengthening phase of the alloy, and how rapidly that phase precipitates out of the solution at elevated temperatures. With all the alloys in this study, the strengthening phases grow from GP zones that prefer nucleation on particles that have already precipitated out of solution. In chromium containing aluminum alloys, GP zones will want to nucleate on the chromium dispersoids within the matrix [17]. The compositions of the alloys cast (Table 3-2) show that while all the alloys contain zirconium, they also all contain some trace amount of chromium.

However, the hardness data does not match the expected trends. The hardness values from zero to two inches follow a seeming parabolic trend, where they increase until the middle of the bar. After the mid-point, the hardness data levels out and starts to decline again around the three inch mark. This second half of the data follows the trends expected and the only data that follows trends seen in literature as a whole are the 7075 samples.

Factorial analysis of the hardness data showed that while the mold type was not significant, the distance from the quenched end was significant with alpha $>0.05$. The data was then separated by alloy and fitted line regressions plots were created, showing the trends in the data clearly. From them, several conclusions could be drawn. While most of the alloys behave in a parabolic manner, 7075 behaves differently. 7075 behaves the way the literature predicts that it would behave [14] (Figure 5-1), as the quench sensitivity of the alloy is apparent, with the hardness decreasing along the length of the bar. The importance of the composition of the alloys as well as the solidification rate is shown here, as other phases like chromium phases or zirconium phases, will have a different morphology depending on the solidification rate. As the morphology, or more specifically, the distribution of these phases throughout the casting changes with solidification rate, the quench sensitivity will be affected locally by the phases present. As solidification rate increases, the expected trend is for hardness to increase as well. The smaller grains and finer distribution of both strengthening phases and primary phases will positively affect the hardness. 


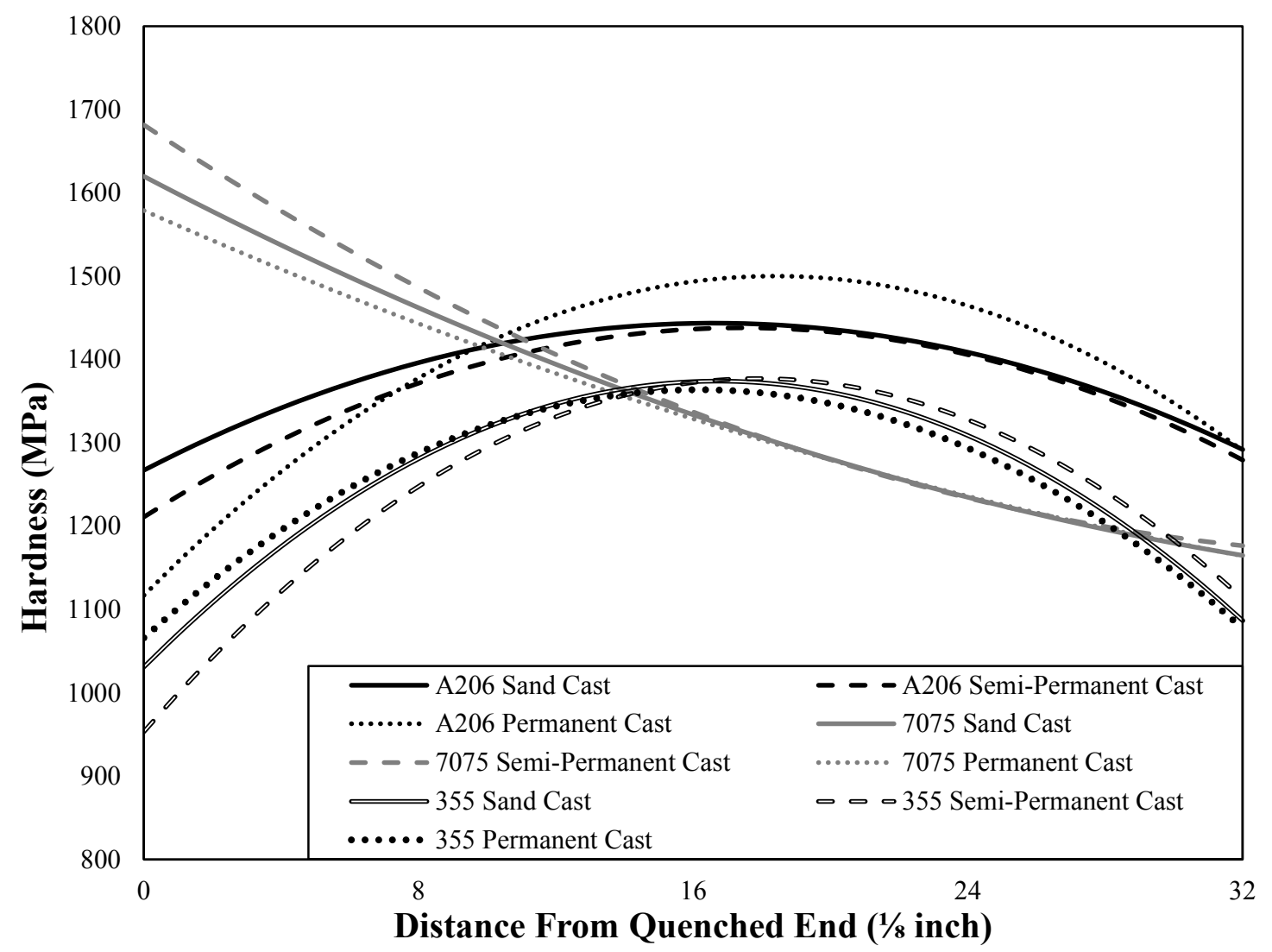

Figure 5-2: Statistical models for A206, 7075 and 319, based off of hardness as a function of distance

The declines and odd trends in the hardness data could have been explained, or at least validated, if the electrical conductivity data did not follow the trends expected in literature. However, the electrical conductivity increases down the length of the bar, as more solute comes out of solution as quenched induced precipitates. If anything, the electrical data matching the trends expected in literature validates the quench rates of the samples.

Comparing the tensile results to the hardness results, the results show why hardness is better at measuring the intrinsic strength of the alloy. Hardness testing is locally done, under compressive loading. This loading and the localization of the test make hardness testing, and the results that come from it, less influenced by other phases within the alloy. Tensile tests, on the other hand, are more influenced by other phases and porosity in the alloy.

Future experiments looking into the quench rate analysis could focus on even more tightly controlled methods, specifically: water temperature regulation, water flow rate control, more thermocouples placed in the samples, and better transfer procedures from the furnace to the JEQ testing apparatus. Better sample creation would also mitigate 
many issues, as tighter process controls will fix many casting issues. SEM and x-ray energy dispersive spectrometry on the samples will show any issues with segregation within the microstructure and TEM studies of the morphology of the precipitates after the quenching and after the aging will shed some light into the kinetics of each individual strengthening phase.

\section{Conclusions}

\subsection{Solidification}

The SDAS results agrees with trends seen in other studies, with the SDAS increasing as solidification rate decreased $[1,2]$. Thee 355 alloy had a SDAS measurement in the sand mold with $48.5 \mu \mathrm{m}$ and a SDAS measurement in the permanent mold with $18 \mu \mathrm{m}$. The porosity data follows literary trends for A206 and 7075, while the other three alloys behave differently [1]. 319 and A356 follow an inverse trend with 355 having a spike in porosity with the semi-permanent mold. The expected trend for these castings was that as the solidification rate decreased, the shrinkage porosity would increase. The highest amount of porosity was seen in the 7075 permanent cast sample, with $9.8 \%$ porosity. The porosity issues can be traced back to a poorly controlled casting process.

Tensile testing of the bars showed internal flaws from casting, proving that the casting process could be refined to get better results, with 7075 needing to be retested. Shrinkage from the casting process effected the testing. While tensile testing does give some idea of what the alloys strength will be, the better test method is hardness testing.

\subsection{Quenching}

Hardness data from the JEQ bars only followed expected trends for 7075 [14], with the rest behaving parabolically. The electrical conductivity data that was collected from the bars did follow literary trends, where electrical conductivity will increase along the length of the bar as solute precipitates out of solution, convoluting the hardness data results. As such, the temperature data collected from the quench tests will not help explain any issues, as the two sets of results contradict each other for certain alloys. The expected trend for the hardness data was to have the hardness be high at the quenched end, where the strengthening phases being locked in perfectly into the solid solution for subsequent aging. The hardness was then to decrease along the length of the sample moving away from the quenched end. The results from the hardness tests show a parabolic nature for every alloy except 7075 . This parabolic nature may be an artifact of improper quenching practices, or incorrect heat treatment, or bad microstructure at the end of the bar. Further investigation was deemed necessary to explain the data. 


\section{References}

1. Kaufman, J.G., E.L. Rooy, and A.F. Society, Aluminum Alloy Castings: Properties, Processes, and Applications. 2004: ASM International.

2. Rundman, K.B., PRINCIPLES of METAL CASTING Textbook. Michigan Technological University.

3. Callister, W.D. and D.G. Rethwisch, Materials Science and Engineering: An Introduction, 8th Edition. 2009: Wiley.

4. Davis, J.R., J.R.D. Associates, and A.S.M.I.H. Committee, Aluminum and Aluminum Alloys. 1993: ASM International.

5. Liu, K., X. Cao, and X.G. Chen, Tensile Properties of Al-Cu 206 Cast Alloys with Various Iron Contents. Metallurgical and Materials Transactions A, 2014. 45(5): p. 2498-2507.

6. You, J.-h., et al., Influence of quench transfer time on microstructure and mechanical properties of 7055 aluminum alloy. Journal of Central South University of Technology, 2008. 15(2): p. 153-158.

7. Zhang, Z.-H., et al., Changes of microstructure of different quench sensitivity 7,000 aluminum alloy after end quenching. Rare Metals, 2014. 33(3): p. 270-275.

8. Toda, H., et al., Influence of high-temperature solution treatments on mechanical properties of an Al-Si-Cu aluminum alloy. Acta Materialia, 2010. 58(6): p. 20142025.

9. Boileau, J.M. and J.E. Allison, The effect of solidification time and heat treatment on the fatigue properties of a cast 319 aluminum alloy. Metallurgical and Materials Transactions A, 2003. 34(9): p. 1807-1820.

10. Campbell, F.C., Phase Diagrams: Understanding the Basics. 2012: ASM International.

11. Shabestari, S.G. and F. Shahri, Influence of modification, solidification conditions and heat treatment on the microstructure and mechanical properties of A356 aluminum alloy. Journal of Materials Science, 2004. 39(6): p. 2023-2032.

12. Talamantes-Silva, M.A., et al., Effect of Solidification Rate and Heat Treating on the Microstructure and Tensile Behavior of an Aluminum-Copper Alloy.

Metallurgical and Materials Transactions B, 2008. 39(6): p. 911-919.

13. Ardell, A.J., Precipitation hardening. Metallurgical Transactions A, 1985. 16(12): p. 2131-2165.

14. Newkirk, J.W. and D.S. MacKenzie, The Jominy end quench for light-weight alloy development. Journal of Materials Engineering and Performance, 2000. 9(4): p. 408-415.

15. Test Methods for Determining Hardenability of Steel. ASTM International.

16. Ma, S., et al., A Methodology to Predict the Effects of Quench Rates on Mechanical Properties of Cast Aluminum Alloys. Metallurgical and Materials Transactions B, 2007. 38(4): p. 583-589.

17. Wagner, J.A. and R.N. Shenoy, The effect of copper, chromium, and zirconium on the microstructure and mechanical properties of $\mathrm{Al}-\mathrm{Zn}-\mathrm{Mg}-\mathrm{Cu}$ alloys. Metallurgical Transactions A, 1991. 22(11): p. 2809-2818. 
18. Starink, M.J. and X.M. Li, A model for the electrical conductivity of peak-aged and overaged $\mathrm{Al}-\mathrm{Zn}-\mathrm{Mg}-\mathrm{Cu}$ alloys. Metallurgical and Materials Transactions A, 2003. 34(4): p. 899-911.

19. Liu, S.D., et al., Influence of aging on quench sensitivity effect of 7055 aluminum alloy. Materials Characterization, 2008. 59(1): p. 53-60.

20. Committee, A.I.H., Properties and Selection: Nonferrous Alloys and SpecialPurpose Materials. 1990: ASM International.

21. Test Methods for Tension Testing of Metallic Materials. ASTM International.

22. Totten, G.E., K. Funatani, and L. Xie, Handbook of Metallurgical Process Design. 2004: CRC Press.

\section{Appendix A: Additional results}

Table 8-1: Results from SDAS measurements

\begin{tabular}{|c|c|c|c|}
\hline Alloy & Sand Mold & Semi-Permanent Mold & Permanent Mold \\
\hline A206 & $63.8 \pm 1.95 \mu \mathrm{m}$ & $34.6 \pm 1.23 \mu \mathrm{m}$ & $29.4 \pm 1.85 \mu \mathrm{m}$ \\
\hline $\mathbf{7 0 7 5}$ & $46.0 \pm 3.14 \mu \mathrm{m}$ & $30 \pm 1.36 \mu \mathrm{m}$ & $25.5 \pm .91 \mu \mathrm{m}$ \\
\hline $\mathbf{3 1 9}$ & $\mathrm{N} / \mathrm{A}$ & $26.8 \pm 1.13 \mu \mathrm{m}$ & $19.4 \pm .99 \mu \mathrm{m}$ \\
\hline $\mathbf{3 5 5}$ & $48.5 \pm 1.56 \mu \mathrm{m}$ & $28.9 \pm .99 \mu \mathrm{m}$ & $18 \pm .79 \mu \mathrm{m}$ \\
\hline $\mathbf{A 3 5 6}$ & $62.1 \pm 3.1 \mu \mathrm{m}$ & $36.4 \pm 1.06 \mu \mathrm{m}$ & $23.4 \pm .81 \mu \mathrm{m}$ \\
\hline
\end{tabular}

Table 8-2: Porosity of samples shown as a percentage difference between literary density and measured density [20]

\begin{tabular}{|l|l|r|r|}
\hline Alloy & Mold & Density & \% Porosity \\
\hline \multirow{3}{*}{ A206 } & Sand & 2.743 & 2.036 \\
\cline { 2 - 4 } & Semi-Perm. & 2.664 & 4.857 \\
\cline { 2 - 4 } & Permanent & 2.629 & 6.107 \\
\hline \multirow{3}{*}{$\mathbf{7 0 7 5}$} & Sand & 2.646 & 5.836 \\
\cline { 2 - 4 } & Semi-Perm. & 2.642 & 5.979 \\
\cline { 2 - 4 } & Permanent & 2.532 & 9.893 \\
\hline \multirow{3}{*}{319} & Sand & 2.611 & 6.416 \\
\cline { 2 - 4 } & Semi-Perm. & 2.659 & 4.695 \\
\cline { 2 - 4 } & Permanent & 2.686 & 3.728 \\
\hline \multirow{3}{*}{355} & Sand & 2.6 & 4.059 \\
\cline { 2 - 4 } & Semi-Perm. & 2.517 & 7.122 \\
\cline { 2 - 4 } & Permanent & 2.595 & 4.244 \\
\hline \multirow{3}{*}{$\mathbf{3 5 6}$} & Sand & 2.493 & 6.629 \\
\cline { 2 - 4 } & Semi-Perm. & 2.542 & 4.794 \\
\cline { 2 - 4 } & Permanent & 2.61 & 2.247 \\
\hline
\end{tabular}




\section{Appendix B: Selected SDAS images for all alloys}
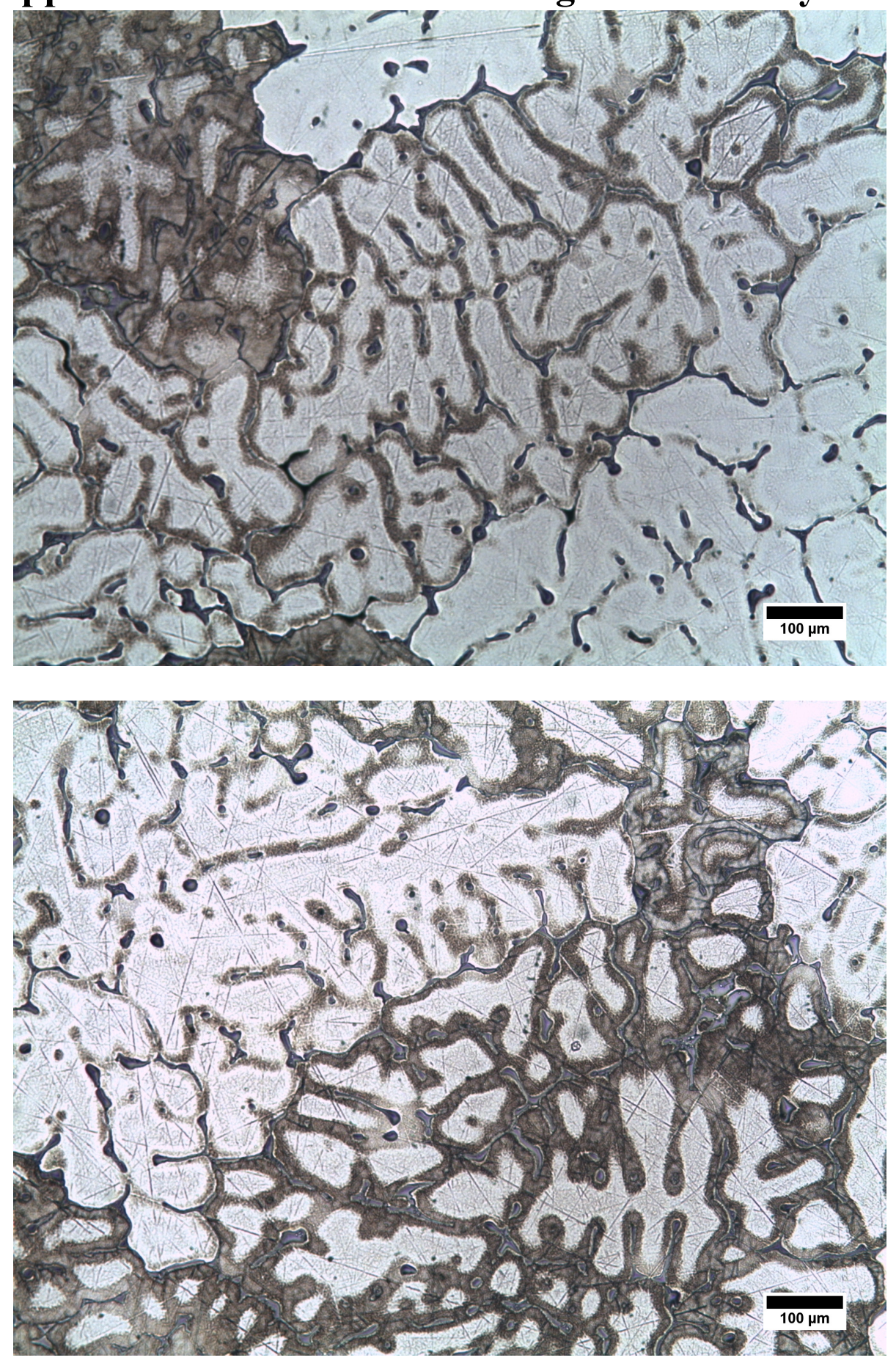

A

Figure 9-1: A206 sand cast micrographs at 100x magnification, etched with Keller's Reagent, at two different locations 

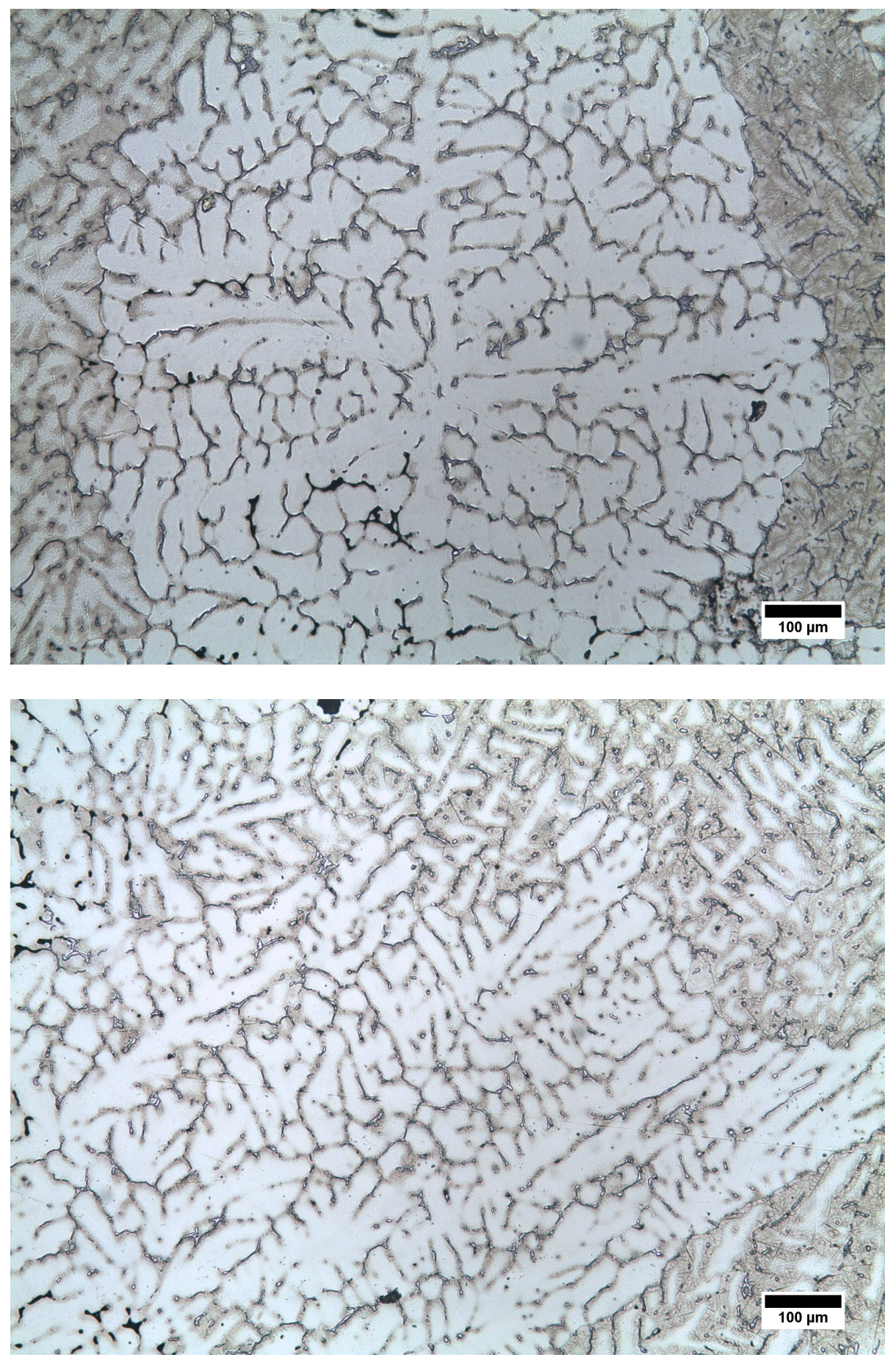

A

B

Figure 9-2:A206 semi-permanent cast micrographs at 100x magnification, etched with Keller's Reagent, at two different locations 


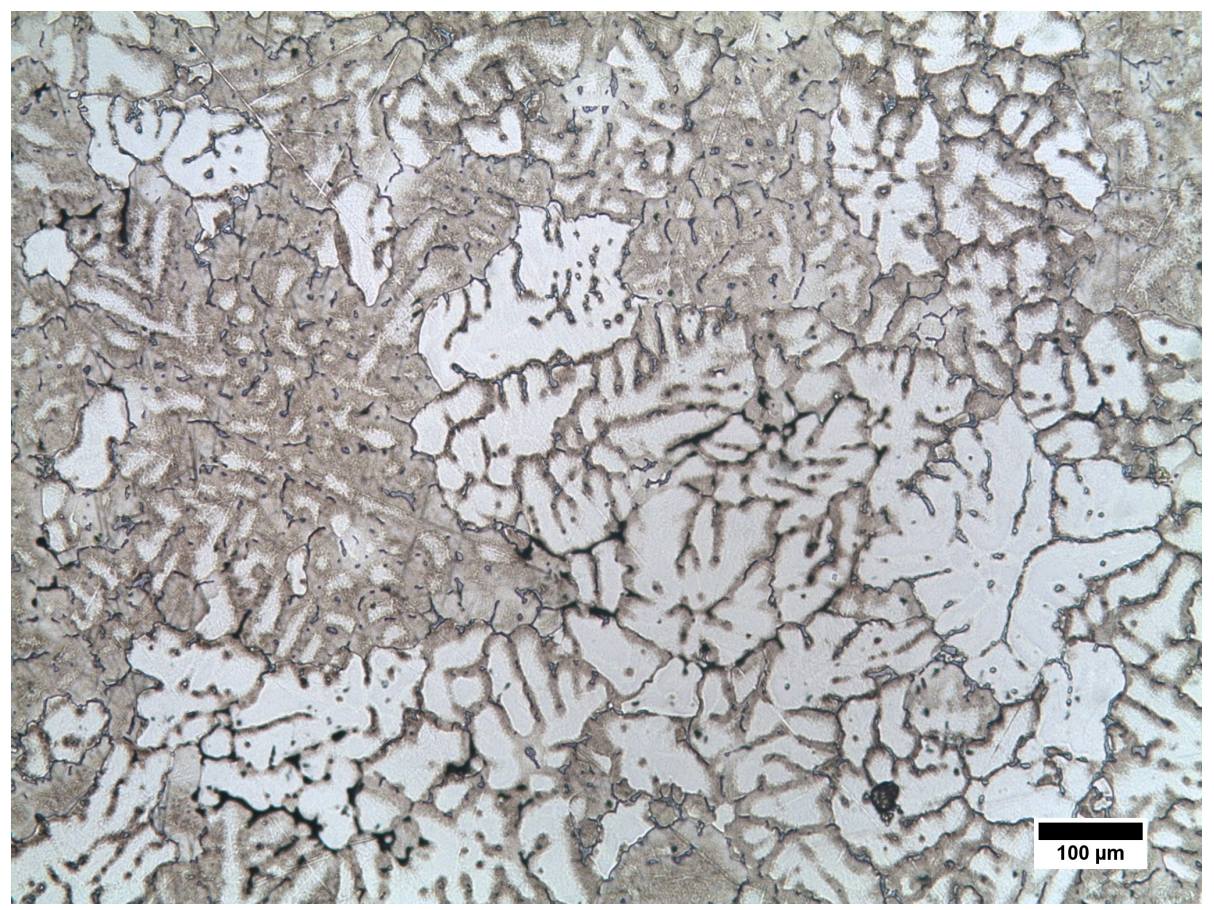

A

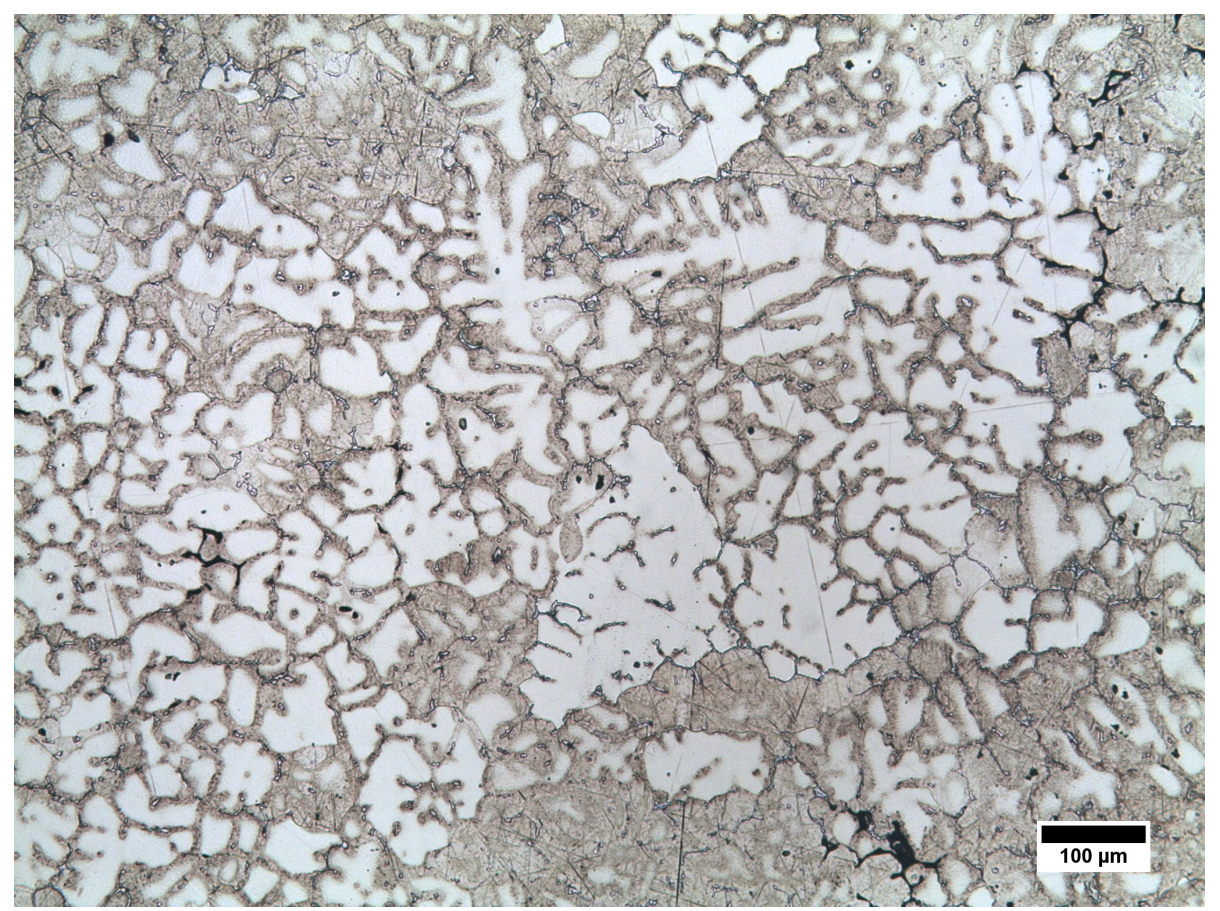

B

Figure 9-3:A206 permanent cast micrographs at 100x magnification, etched with Keller's Reagent, at two different locations 

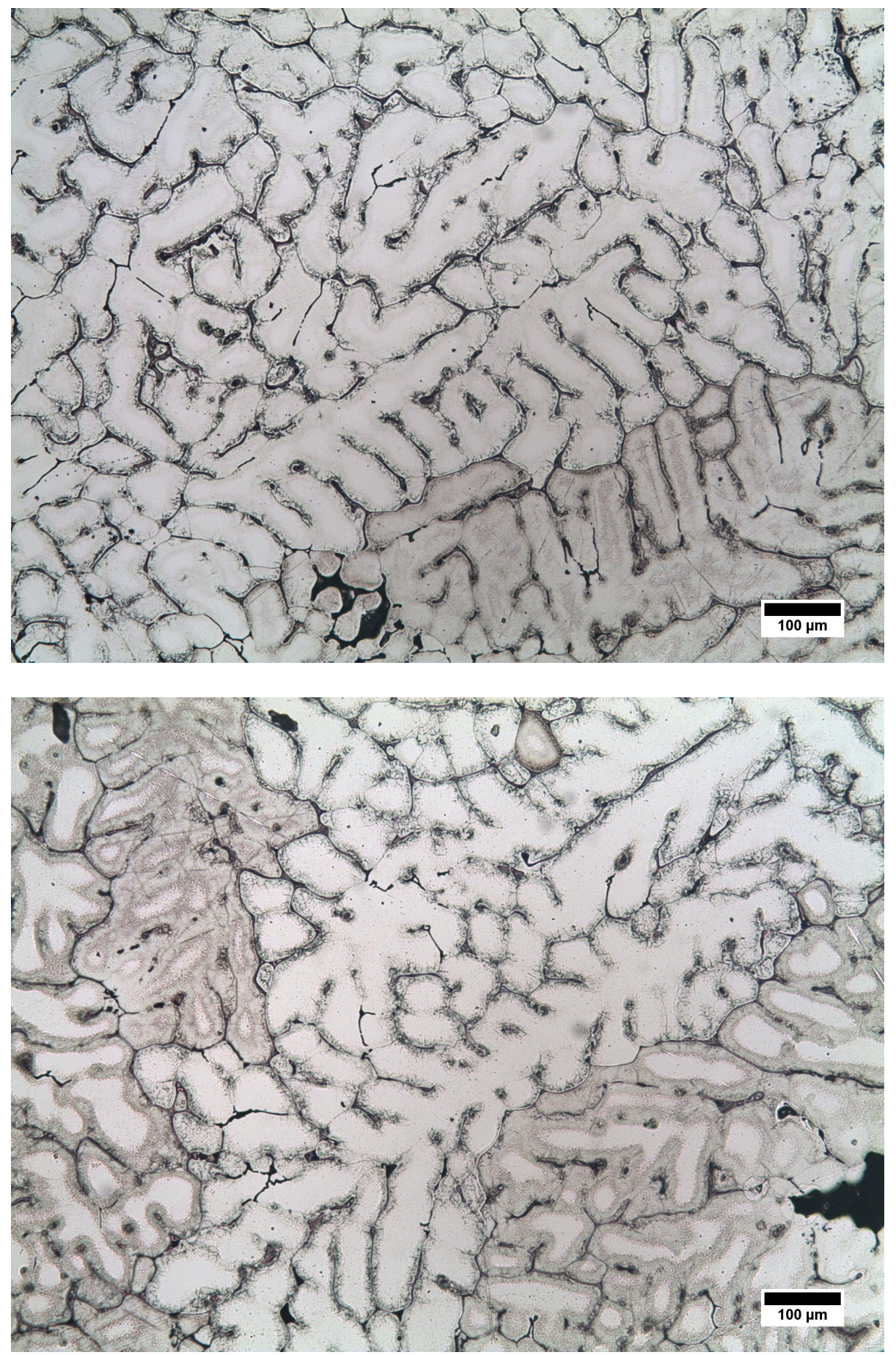

A

B

Figure 9-4:: 7075 sand cast micrographs at 100x magnification, etched with Keller's Reagent, at two different locations 


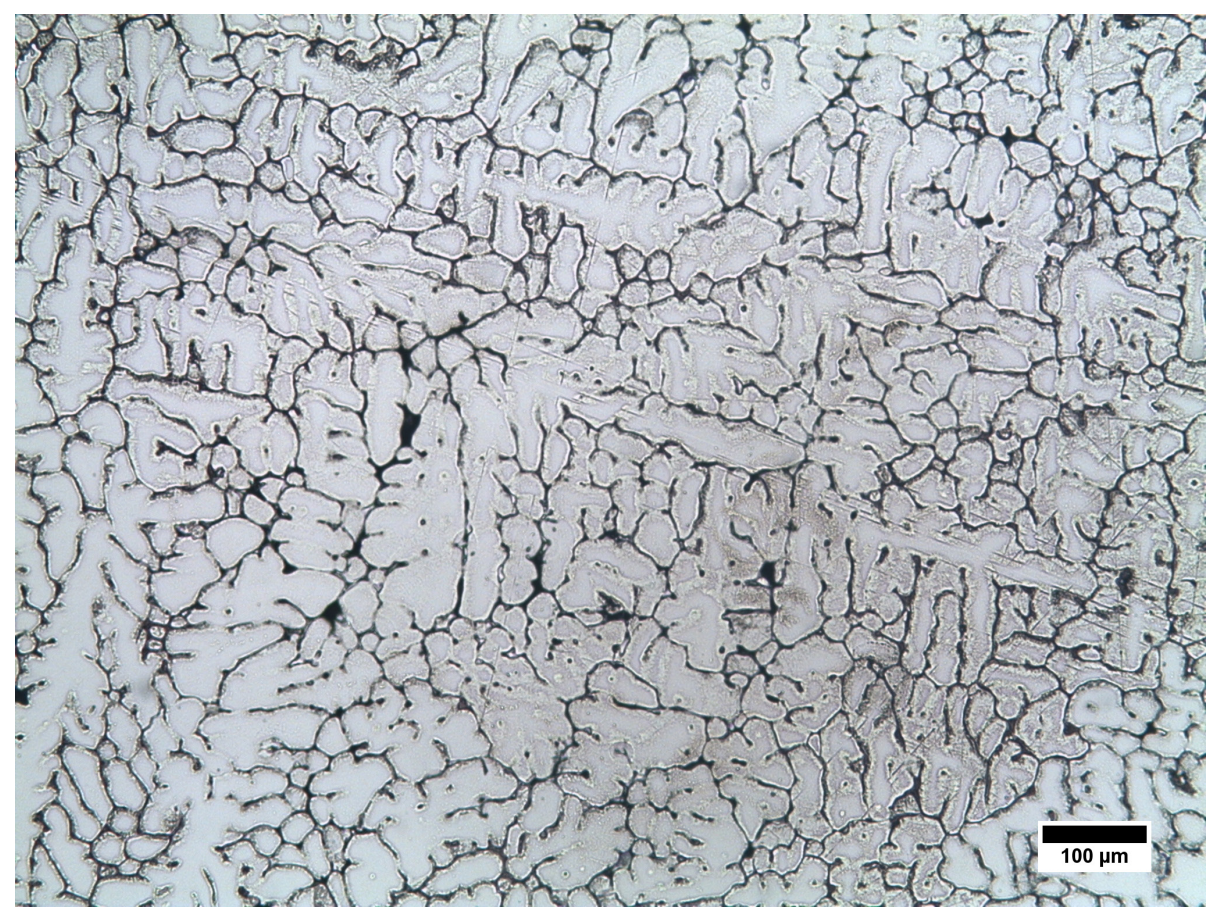

A

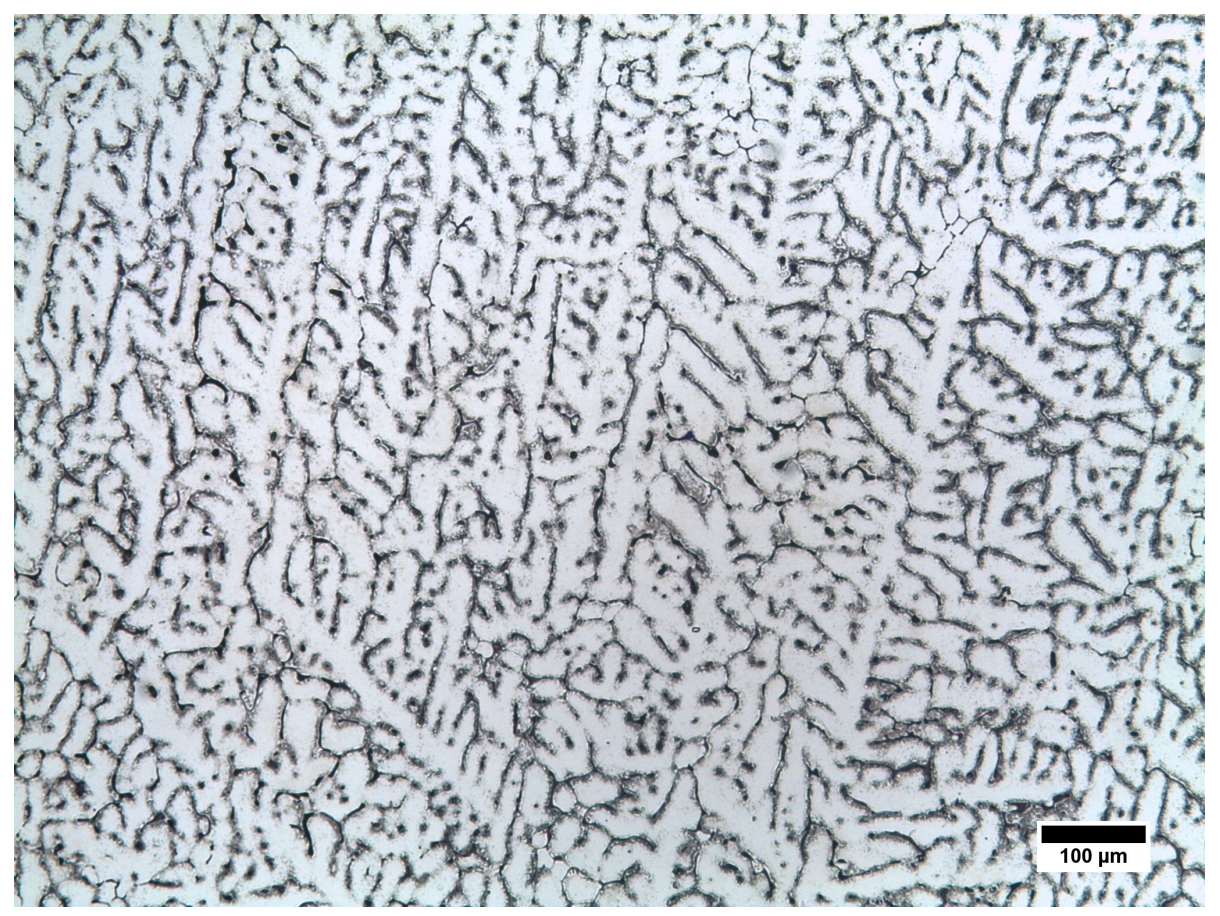

B

Figure 9-5:7075 semi-permanent cast micrographs at 100x magnification, etched with Keller's Reagent, at two different locations 

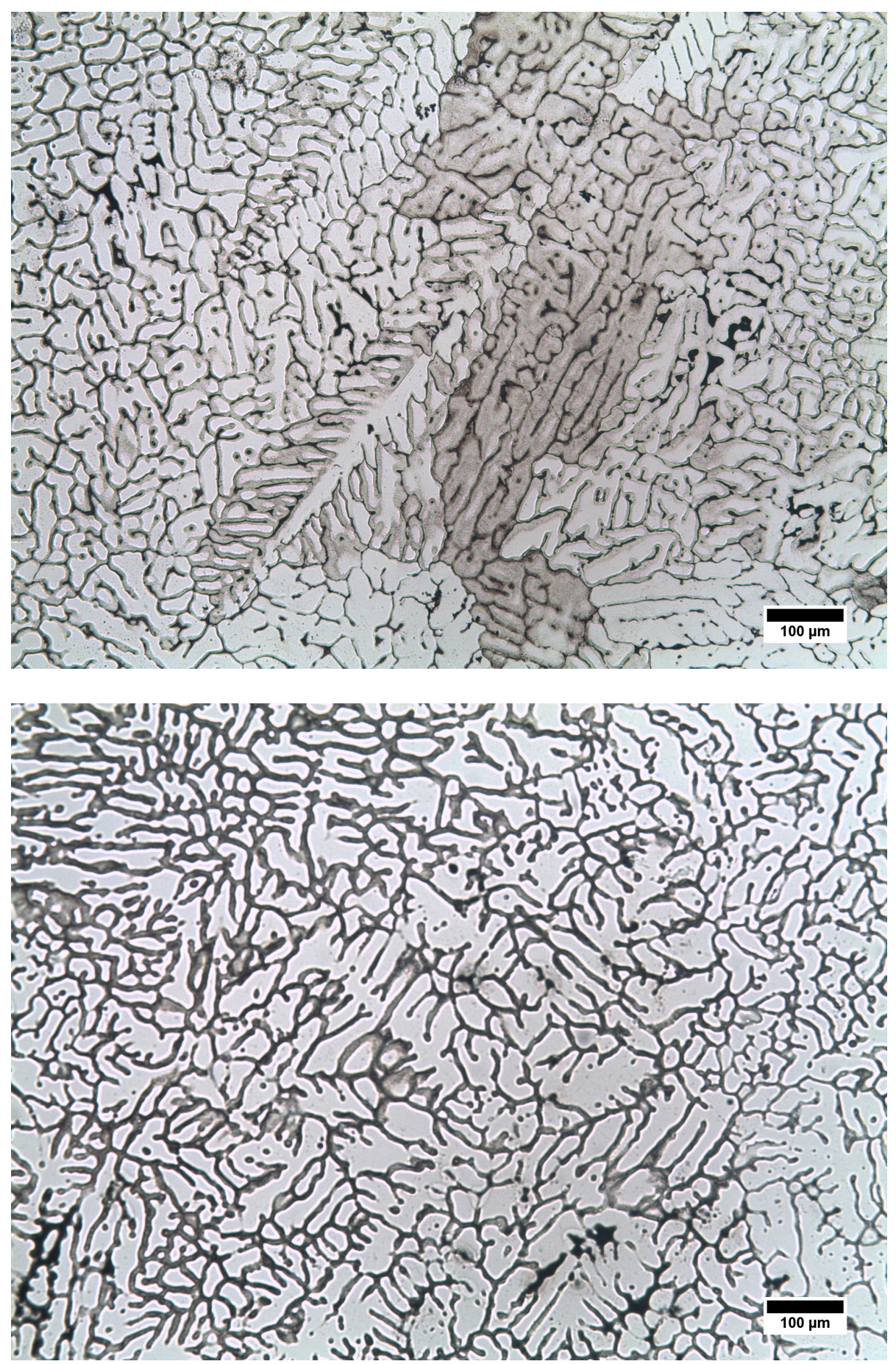

Figure 9-6:7075 permanent cast micrographs at 100x magnification, etched with Keller's Reagent, at two different locations 

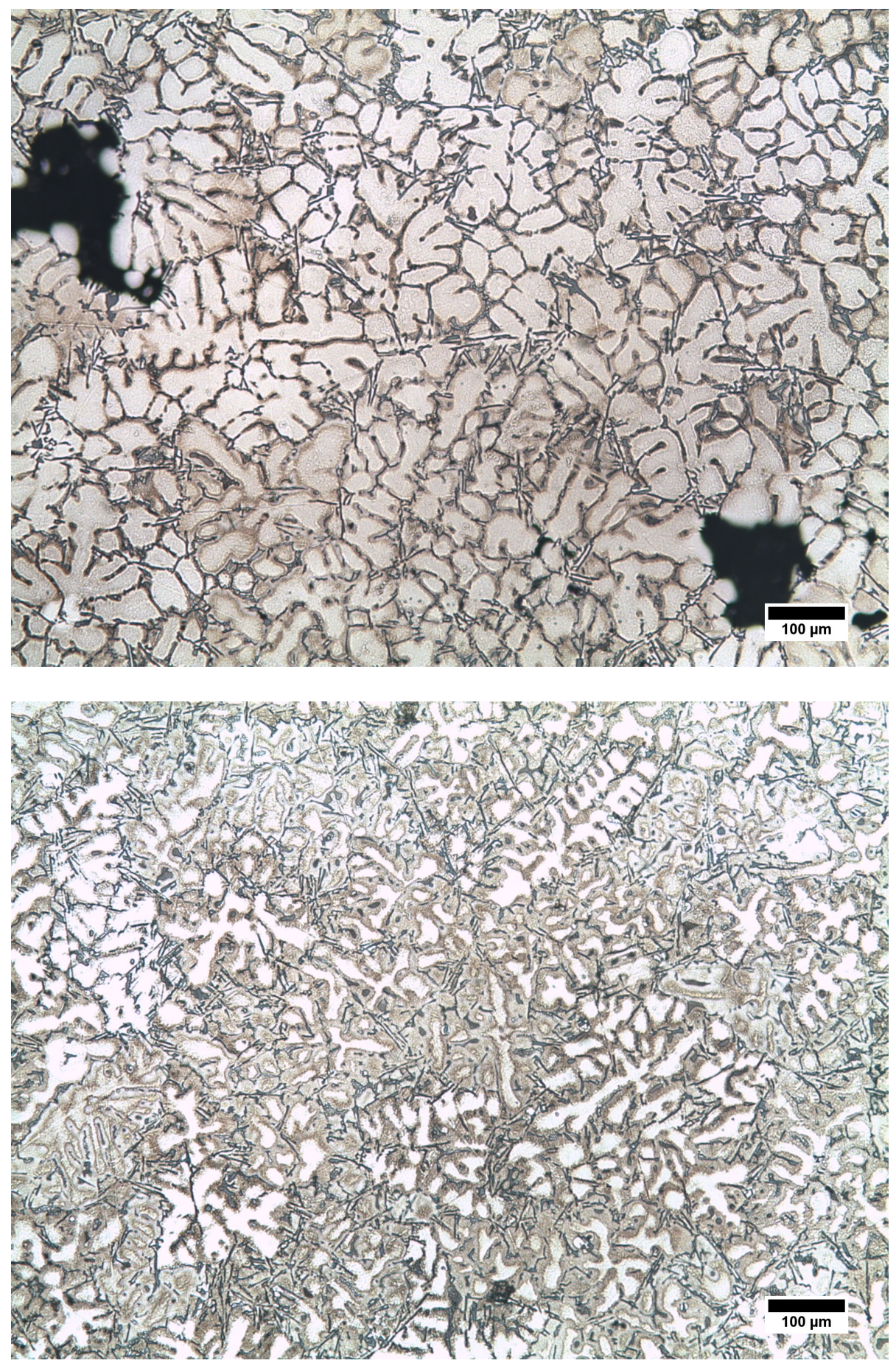

Figure 9-7:319 semi-permanent cast micrographs at 100x magnification, etched with Keller's Reagent, at two different locations 

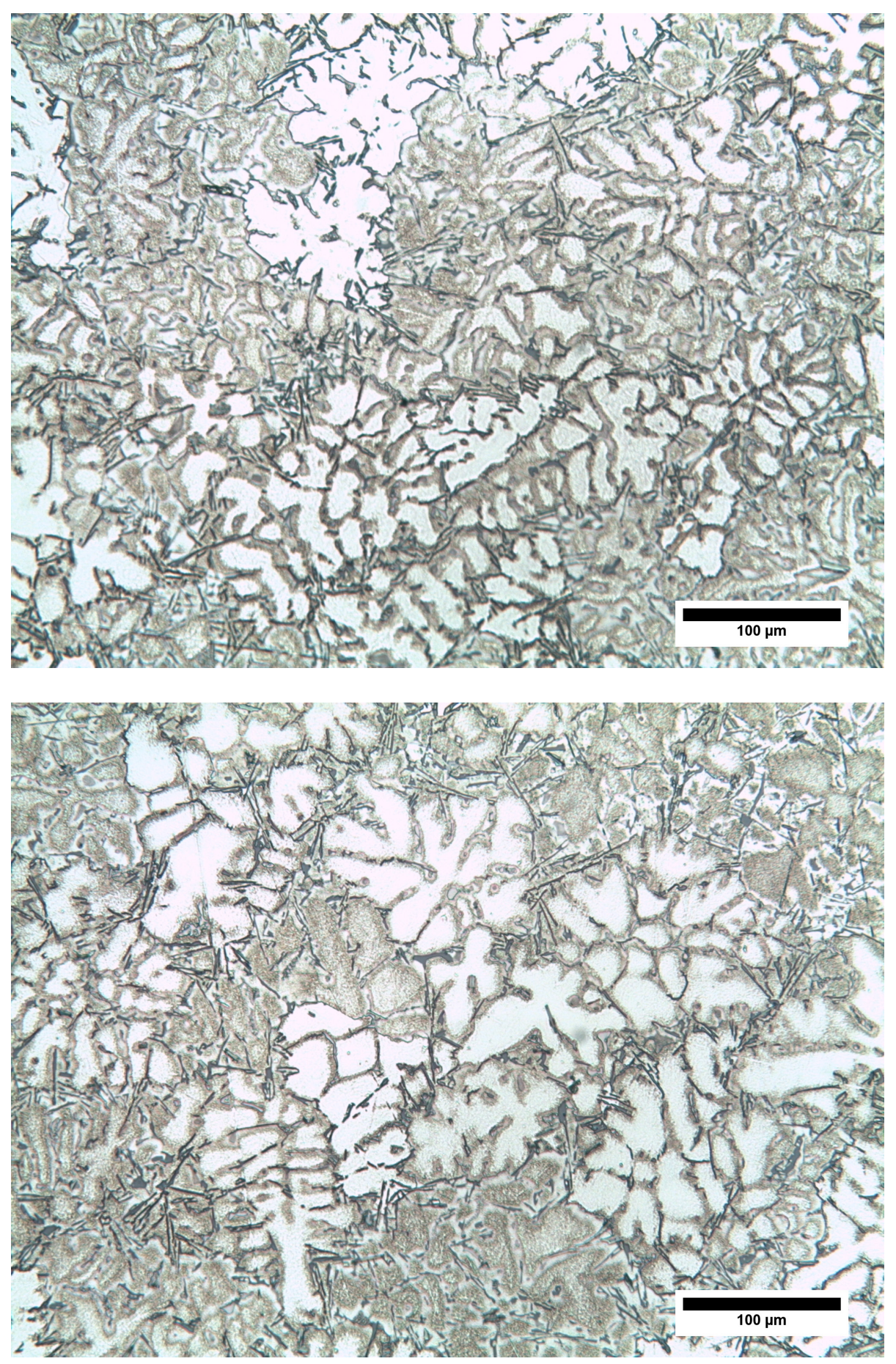

A

B

Figure 9-8:319 permanent cast micrographs at 200x magnification etched with Keller's Reagent, at two different locations 

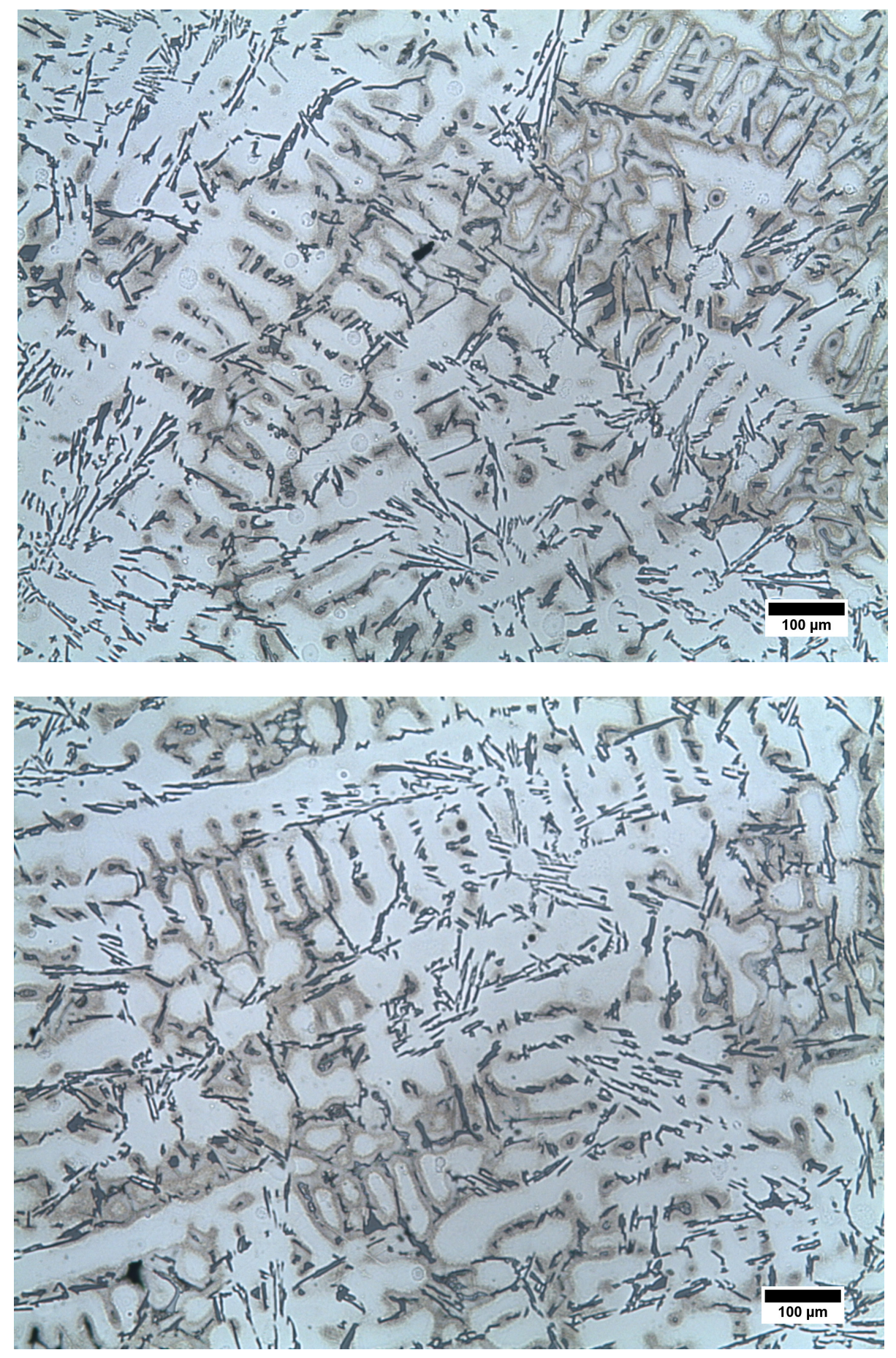

A

B

Figure 9-9:355 sand cast micrographs at 100x magnification etched with Keller's Reagent, at two different locations 

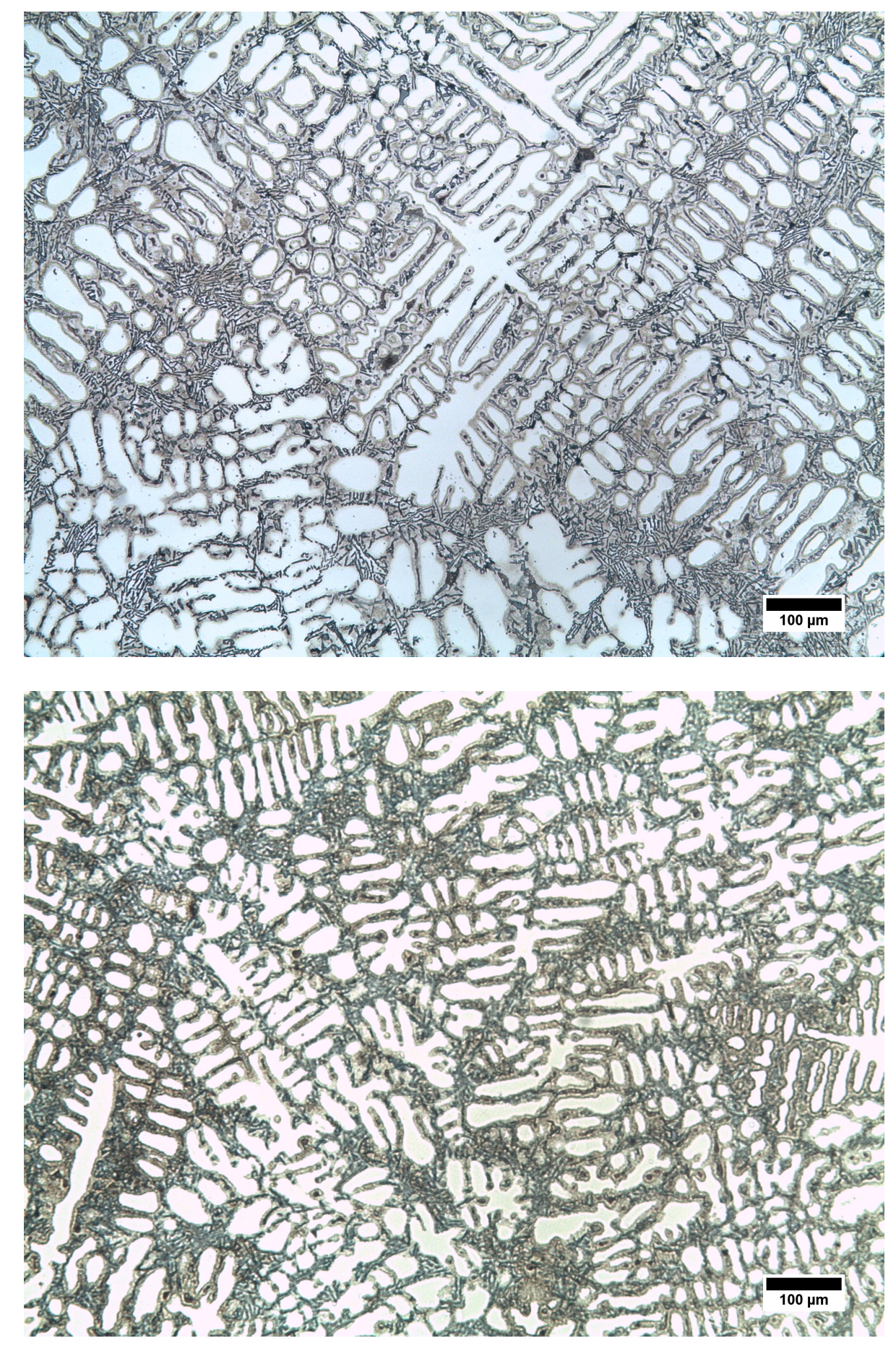

Figure 9-10:355 semi-permanent cast micrographs at 100x magnification etched with Keller's Reagent, at two different locations 

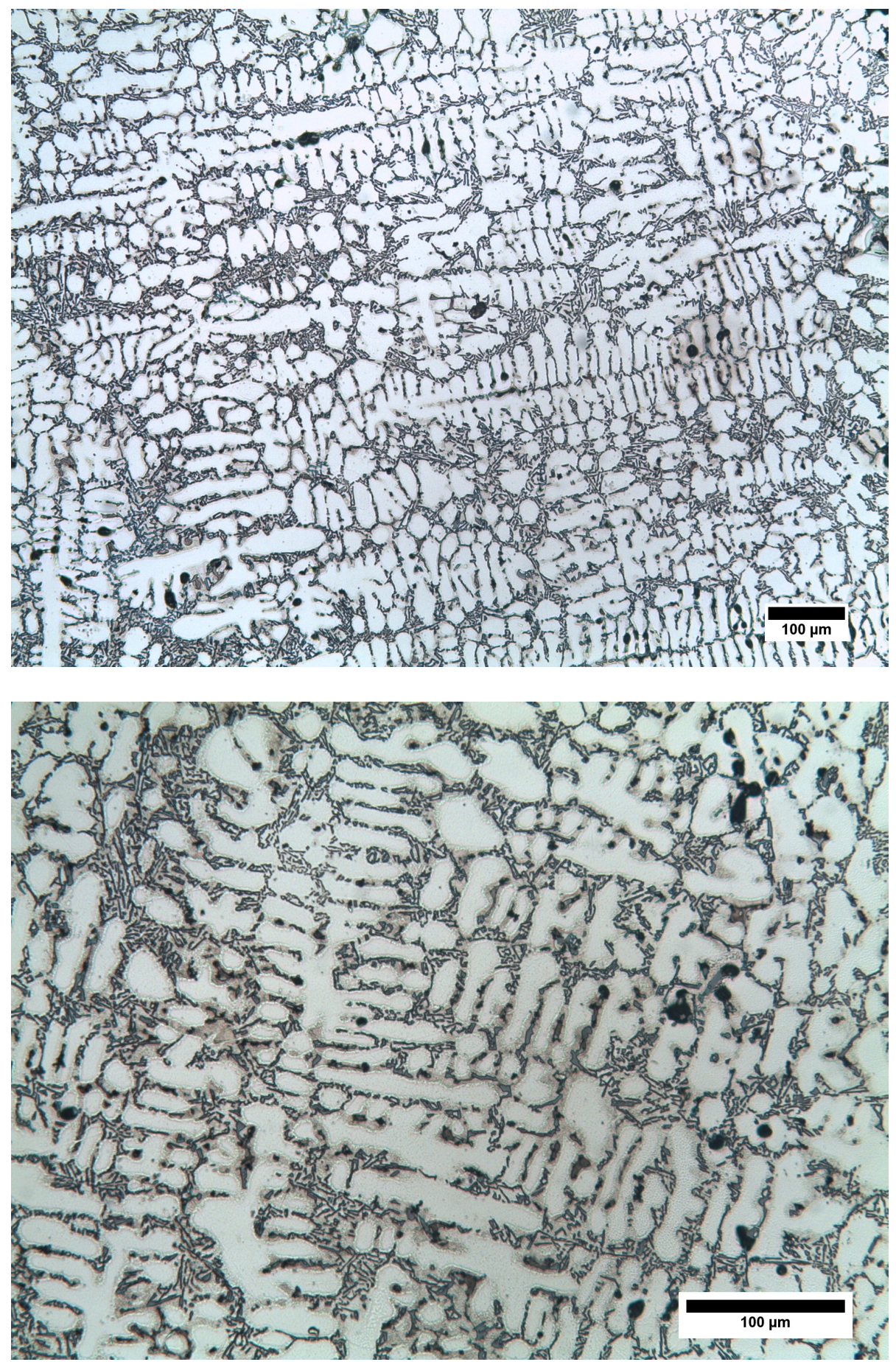

A

Figure 9-11:355 permanent cast micrographs at a) 100x magnification and b) 200x magnification etched with Keller's Reagent, at two different locations 

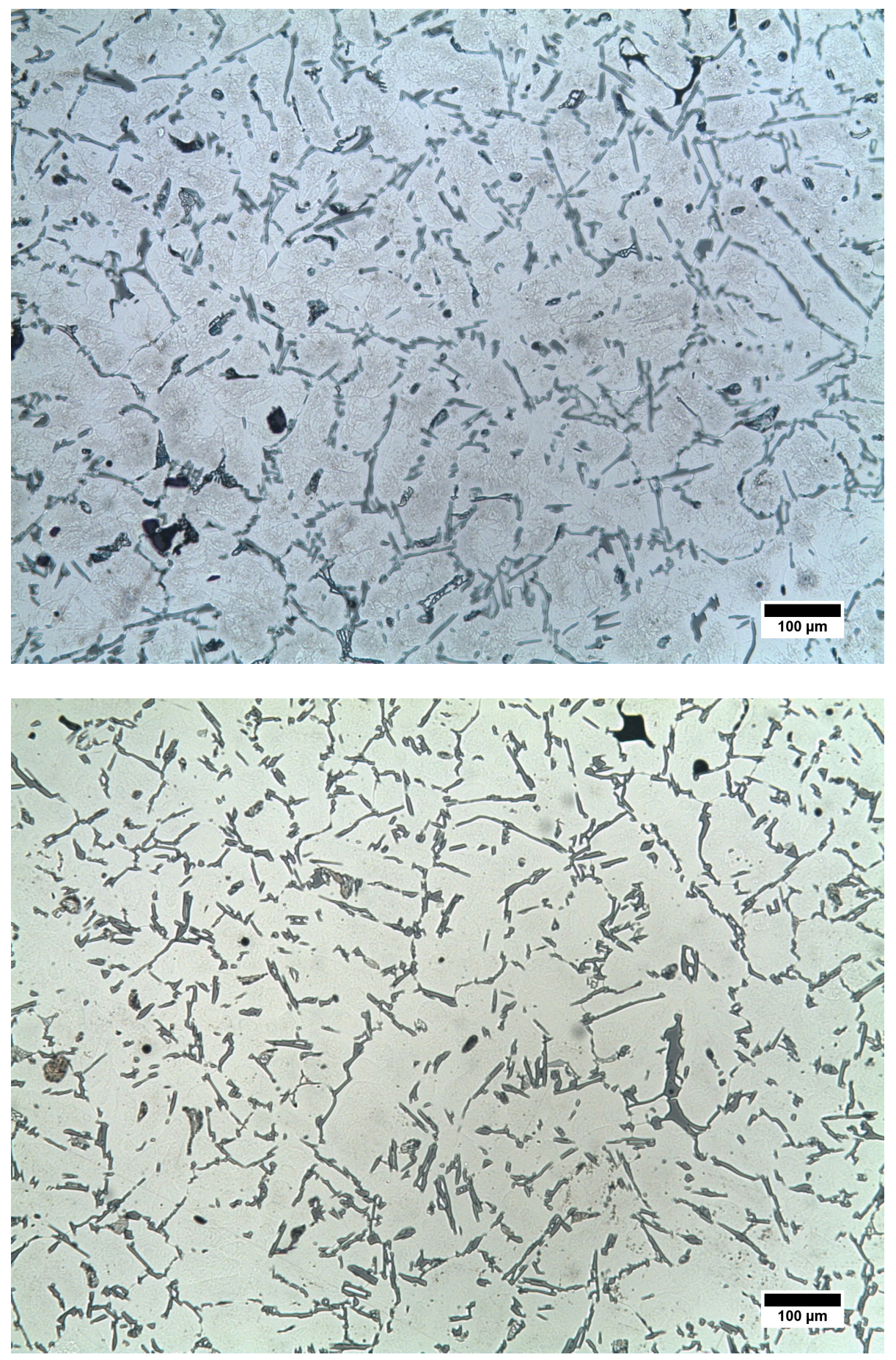

Figure 9-12:A356 sand cast micrographs at 100x magnification etched with Keller's Reagent at two different locations 

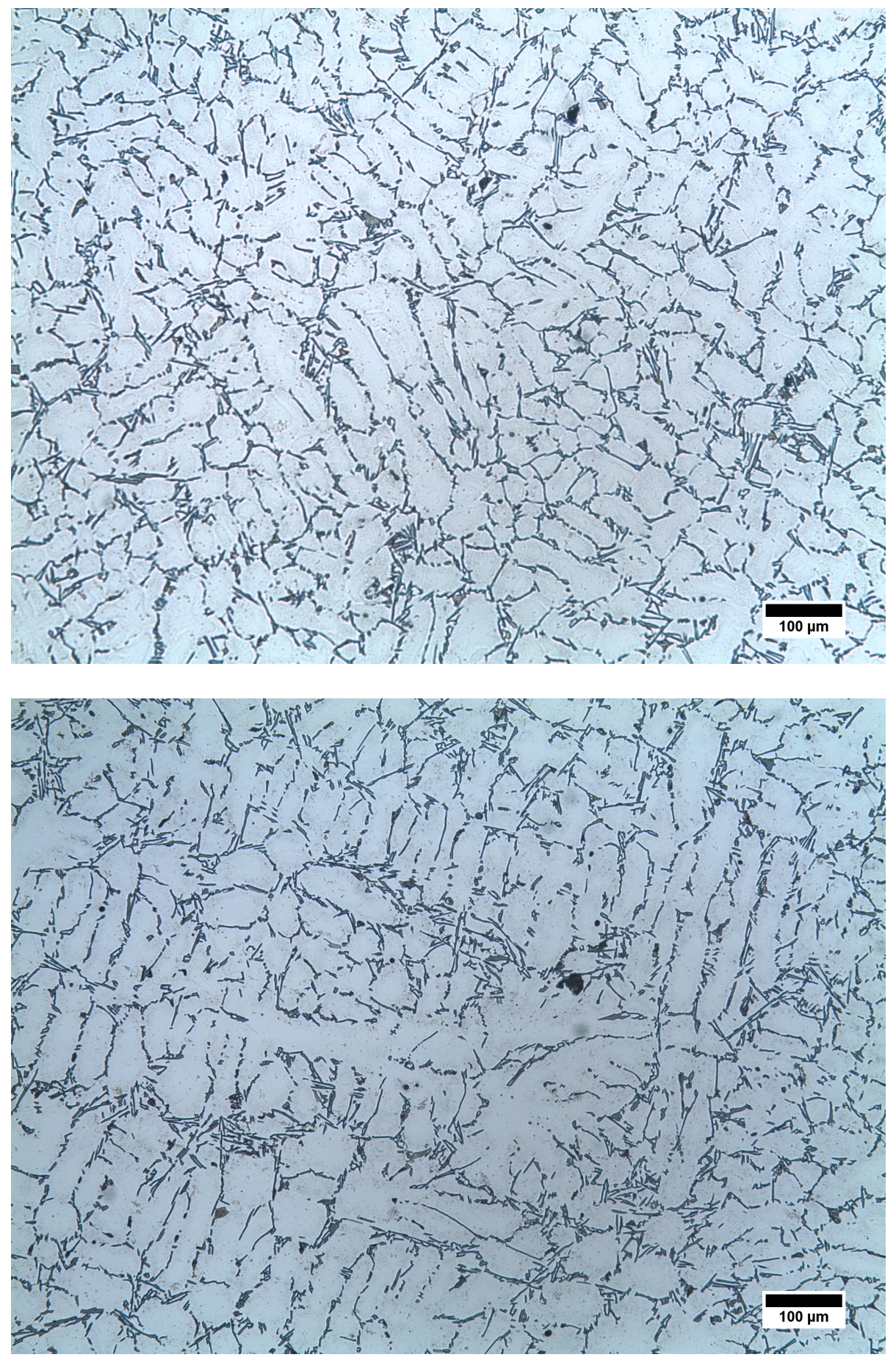

Figure 9-13:A356 semi-permanent micrographs at 100x magnification etched with Keller's Reagent at two different locations 

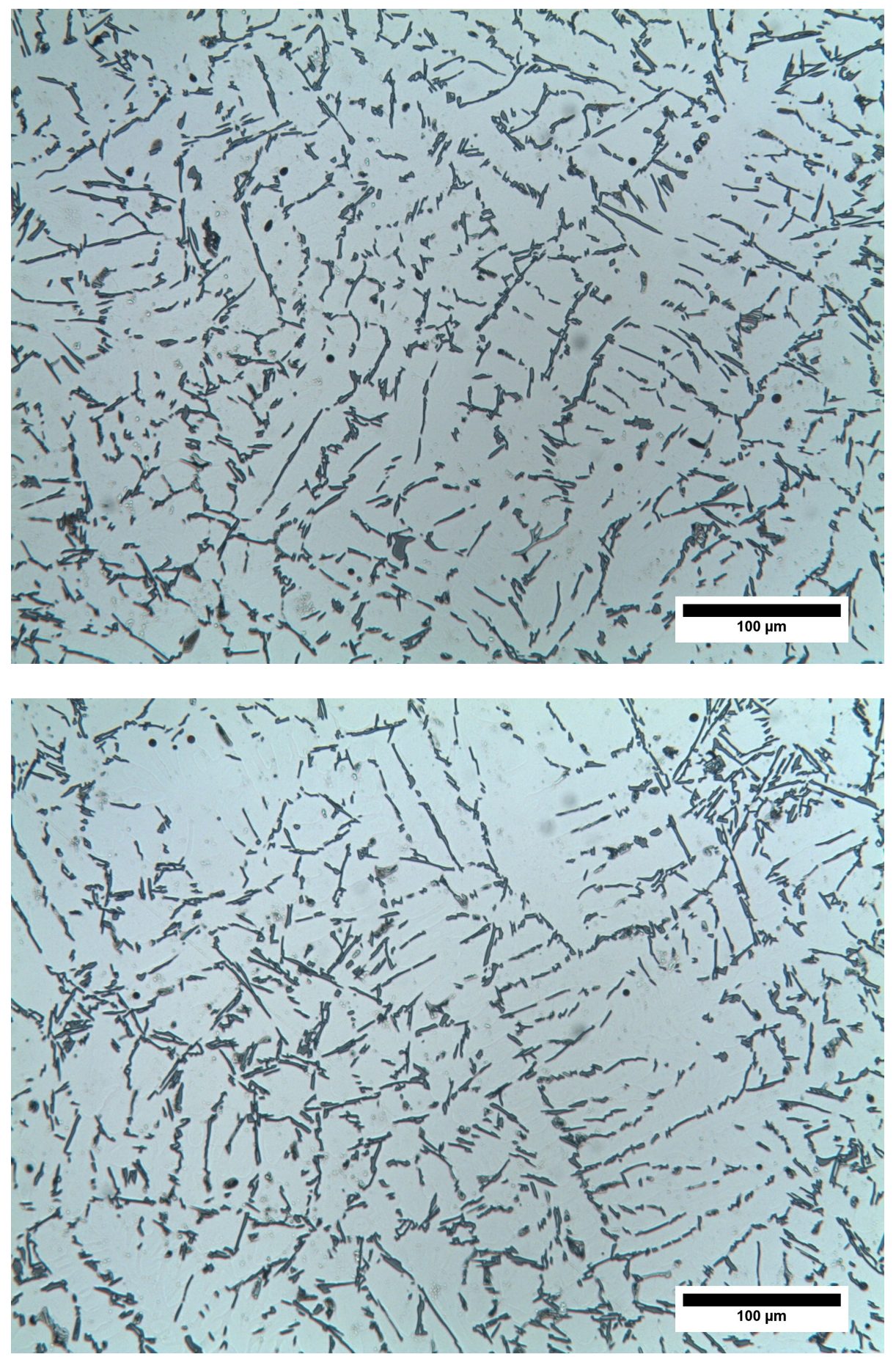

Figure 9-14:A356 permanent cast micrographs at 200x magnification etched with Keller's Reagent, at two different locations 